Алгебра и анализ

Tом 22 (2010), № 5
St. Petersburg Math. J. Vol. 22 (2011), No. 5, Pages 703-736 S 1061-0022(2011)01164-1

Article electronically published on June 27, 2011

\title{
SPECTRAL ESTIMATES FOR A PERIODIC FOURTH-ORDER OPERATOR
}

\author{
A. V. BADANIN AND E. L. KOROTYAEV
}

\begin{abstract}
The operator $H=\frac{d^{4}}{d t^{4}}+\frac{d}{d t} p \frac{d}{d t}+q$ with periodic coefficients $p, q$ on the real line is considered. The spectrum of $H$ is absolutely continuous and consists of intervals separated by gaps. The following statements are proved: 1) the endpoints of gaps are periodic or antiperiodic eigenvalues or branch points of the Lyapunov function, and moreover, their asymptotic behavior at high energy is found; 2) the spectrum of $H$ at high energy has multiplicity two; 3) if $p$ belongs to a certain class, then for any $q$ the spectrum of $H$ has infinitely many gaps, and all branch points of the Lyapunov function, except for a finite number of them, are real and negative; 4) if $q=0$ and $p \rightarrow 0$, then at the beginning of the spectrum there is a small spectral band of multiplicity 4 , and its asymptotic behavior is found; the remaining spectrum has multiplicity 2 .
\end{abstract}

\section{§1. INTRODUCTION AND MAIN RESULTS}

Consider a selfadjoint operator $H=\frac{d^{4}}{d t^{4}}+\frac{d}{d t} p \frac{d}{d t}+q$ that acts in $L^{2}(\mathbb{R})$ and has real 1-periodic coefficients $p$ and $q$ such that $p, p^{\prime}, q \in L^{1}(0,1)$. Henceforth we use the notation $f^{\prime}=\partial_{t} f, f^{(k)}=\partial_{t}^{k} f$. It is known that the spectrum of the operator $H$ is absolutely continuous and consists of nondegenerate intervals (see [8, Theorem XIII.7.64]). We introduce the fundamental solutions $\varphi_{j}(t, \lambda), j \in \mathbb{N}_{3}=\{0,1,2,3\}$, of the equation

$$
y^{(\mathrm{iv})}+\left(p y^{\prime}\right)^{\prime}+q y=\lambda y, \quad(t, \lambda) \in \mathbb{R} \times \mathbb{C},
$$

that satisfy the conditions $\varphi_{j}^{(k)}(0, \lambda)=\delta_{j k}, j, k \in \mathbb{N}_{3}$, where $\delta_{j k}$ is the Kronecker symbol. We define a $4 \times 4$ monodromy matrix $M$ and the characteristic polynomial $D$ by the relations

$$
M(\lambda)=\left(\varphi_{j}^{(k)}(1, \lambda)\right)_{k, j=0}^{3}, \quad D(\tau, \lambda)=\operatorname{det}\left(M(\lambda)-\tau I_{4}\right), \quad(\tau, \lambda) \in \mathbb{C}^{2} .
$$

It is known that $M$ is an entire matrix-valued function. An eigenvalue $\tau(\lambda)$ of the matrix $M(\lambda)$ is called a multiplier and is a zero of the algebraic equation $D(\tau, \lambda)=0$. The matrix $M$ has four multipliers $\tau_{1}, \tau_{2}, \tau_{3}, \tau_{4}$ (counted with multiplicity), and $\tau_{3}(\lambda)=$ $\tau_{1}^{-1}(\lambda), \tau_{4}(\lambda)=\tau_{2}^{-1}(\lambda)$ for all $\lambda \in \mathbb{C}$ (see 31]). The spectrum of $H$ has the form $\sigma(H)=\left\{\lambda \in \mathbb{C}:\left|\tau_{1}(\lambda)\right|=1\right.$ or $\left.\left|\tau_{2}(\lambda)\right|=1\right\}$.

2010 Mathematics Subject Classification. Primary 34L15; Secondary 34L40.

Key words and phrases. Periodic differential operator, spectral bands, spectral asymptotics.

The work of A. V. Badanin was partially supported by a joint grant of DAAD (the program "Mikhail Lomonosov-2007") and by a grant of the Ministry of Education of RF (the program "Development of the scientific potential of the higher school in 2006-2008"). Part of the paper was written at the Mathematical Institute of Potsdam University, Germany (September-December, 2007). Part of the work was done by E. L. Korotyaev at the Institute of Mathematics of Tsukkuba University, Japan (March, 2010) and at École Polytéchnique, France (April-July, 2010). The authors are grateful to these institutions for their hospitality. 
Consider the entire function

$$
\rho=\frac{T_{2}+1}{2}-T_{1}^{2}, \quad \text { where } \quad T_{\nu}=\frac{1}{4} \operatorname{Tr} M^{\nu}, \quad \nu=1,2 .
$$

The function $\rho$ is real on $\mathbb{R}$ and $\rho(\lambda)>0$ for $\lambda>R$, where $R$ is a real number (see Lemma 3.3). Denote by $\mathscr{R}$ the two-sheeted Riemann surface of the function $\sqrt{\rho}$. We select a single-valued branch of the function $\sqrt{\rho}$ on a cut plane. To this end, we draw cuts along the intervals of the real axis on which $\rho<0$. We consider the zeros of $\rho$ that lie in the upper half-plane, join them pairwise with nonintersecting curves, and draw cuts along these curves. We draw symmetric cuts in the lower half-plane. If the function $\rho$ has an odd number of zeros in the upper half-plane, then we connect the remaining zero to the zero in the lower half-plane conjugate to it with a curve that intersects the real axis at any point where $\rho<0$, and cut the plane along this curve. Denote by $\Lambda$ the plane with such cuts. We fix a branch of the function $\sqrt{\rho}$ in the domain $\Lambda$ by the condition $\sqrt{\rho(\lambda)}>0$ for $\lambda \in(R,+\infty)$.

We state a preliminary result concerning the Lyapunov function. Throughout the paper,

$$
z=\lambda^{\frac{1}{4}}, \quad \arg z \in\left(-\frac{\pi}{4}, \frac{\pi}{4}\right] \quad \text { for } \quad \lambda \in \mathbb{C}, \quad \arg \lambda \in(-\pi, \pi] .
$$

Theorem 1.1. i) The functions $\Delta_{\nu}=\frac{1}{2}\left(\tau_{\nu}+\tau_{\nu}^{-1}\right), \nu=1,2$, are branches of the Lyapunov function $\Delta=T_{1}+\sqrt{\rho}$, which is analytic on the surface $\mathscr{R}$. We fix the branches of $\Delta_{\nu}$ in the domain $\Lambda$ by the relations

$$
\Delta_{\nu}=T_{1}-(-1)^{\nu} \sqrt{\rho}, \quad \nu=1,2 .
$$

Then the following relations are valid:

$$
\begin{gathered}
D(\tau, \cdot)=\operatorname{det}\left(M-\tau I_{4}\right)=\left(\tau^{2}-2 \Delta_{1} \tau+1\right)\left(\tau^{2}-2 \Delta_{2} \tau+1\right), \quad \tau \in \mathbb{C}, \\
\Delta_{1}(\lambda)=\cosh z\left(1+O\left(\frac{1}{z}\right)\right), \quad \Delta_{2}(\lambda)=\cos z\left(1+O\left(\frac{1}{z}\right)\right)
\end{gathered}
$$

as $|\lambda| \rightarrow \infty,|z-\pi n|>1,|z-(1 \pm i) \pi n|>1, n \in \mathbb{Z}$.

ii) Let $\Delta_{\nu}(\lambda) \in(-1,1)$ for a branch $\nu \in\{1,2\}$, and let $\lambda \in \mathbb{R}$ not be a branch point of $\Delta$. Then $\Delta_{\nu}^{\prime}(\lambda) \neq 0$.

Remark. For any $p$ and $q$, the function $\rho$ has at least one zero of odd multiplicity (see Lemma 3.3). Therefore, it cannot be an exact square of an entire function.

Consider the functions $D_{ \pm}=\frac{1}{4} \operatorname{det}\left(M \mp I_{4}\right)=\left(\Delta_{1} \mp 1\right)\left(\Delta_{2} \mp 1\right)$. The zeros of $D_{+}$ (of $D_{-}$) are eigenvalues of the periodic (respectively, antiperiodic) problem. Denote by $\lambda_{0}^{+}, \lambda_{2 n}^{ \pm}, n \geq 1$, the sequence of zeros of $D_{+}$and by $\lambda_{2 n-1}^{ \pm}, n \geq 1$, the sequence of zeros of $D_{-}$enumerated (counting the multiplicities) in such a way that

$$
\begin{aligned}
& \lambda_{0}^{+} \leq \lambda_{2}^{-} \leq \lambda_{2}^{+} \leq \lambda_{4}^{-} \leq \lambda_{4}^{+} \leq \lambda_{6}^{-} \leq \cdots, \\
& \lambda_{1}^{-} \leq \lambda_{1}^{+} \leq \lambda_{3}^{-} \leq \lambda_{3}^{+} \leq \lambda_{5}^{-} \leq \lambda_{5}^{+} \leq \cdots
\end{aligned}
$$

The branch points of the Lyapunov function are zeros of $\rho$ (see (1.3)) of odd multiplicity. A numerical investigation of the function $\rho$ for the operator $H$ with a periodic $\delta$-potential (see [1]) shows that, as the coefficients of equation (1.1) vary continuously, two different branch points may merge and form a zero of even multiplicity of $\rho$, which is no longer a branch point of the Lyapunov function. Conversely, a zero of even multiplicity of $\rho$, which is not a branch point of the Lyapunov function, may split into two zeros of odd multiplicity as the coefficients of equation (1.1) vary continuously, and thus it may generate two branch points of the Lyapunov function. Moreover, branch points of the Lyapunov function may be complex for some values of the coefficients of equation (1.1), 
and they become real, creating a gap in the spectrum of $H$, for some other values of the coefficients. Such a behavior is similar to the behavior of resonances in the scattering problem for the Schrödinger operator, although in reality the resonances in the scattering problem are not related to the branch points of the Lyapunov function for the operator $H$ (for example, see [14, 35]). Nevertheless, in the same way as in [1, 2, 15, 13, 15, we call the zeros of the function $\rho$ resonances. The multiplicity of a resonance is the multiplicity of the corresponding zero.

Example 1. If $p=q=0$, then the functions corresponding to $T_{\nu}, \rho, D_{ \pm}$, and $\Delta$ have the form

$$
\begin{array}{r}
T_{\nu}^{0}=\frac{\cosh \nu z+\cos \nu z}{2}, \quad \rho^{0}=\frac{(\cosh z-\cos z)^{2}}{4}, \quad D_{ \pm}^{0}=(\cos z \mp 1)(\cosh z \mp 1) \\
\Delta^{0}=\cosh \lambda^{\frac{1}{4}}, \quad \Delta_{1}^{0}=\cosh z, \quad \Delta_{2}^{0}=\cos z .
\end{array}
$$

From (1.8) it follows that $\rho^{0} \leq 0$ on $\mathbb{R}_{-}$, and the resonances are equal to $r_{0}^{-, 0}=0, r_{n}^{ \pm, 0}=$ $-4(\pi n)^{4}, n \geq 1$. Moreover, the 2-periodic (i.e., periodic and antiperiodic) eigenvalues are equal to $\lambda_{0}^{+, 0}=0, \lambda_{n}^{ \pm, 0}=(\pi n)^{4}, n \geq 1$. The surface $\mathscr{R}^{0}$ coincides with the Riemann surface of the function $\sqrt{\lambda}$.

Example 2. Consider the operator $H=\widetilde{H}^{2}$, where $\widetilde{H}=-\frac{d^{2}}{d t^{2}}+p$ is the Hill operator on $\mathbb{R}$. Recall that the spectrum $\sigma(\tilde{H})$ is bounded from below and consists of bands separated by gaps. Let $\sigma^{ \pm}=\sigma(\tilde{H}) \cap \mathbb{R}_{ \pm}$(the set $\sigma^{-}$may be empty). The spectrum of $H=\tilde{H}^{2}$ has the form $\sigma(H)=\left\{\lambda \geq 0: \lambda=z^{2}, z \in \sigma_{-} \cup \sigma_{+}\right\}$and has multiplicity 4 at the points where $\sigma_{+}$and $\sigma_{-}$overlap; the remaining part of the spectrum has multiplicity 2.

Many papers (see, e.g., Gel'fand and Lidskiı̆ [10], Gesztesy with coauthors [6, 7], Carlson [3, 4, Korotyaev with coauthors [2, 5, 13, 15, Kreĭn [19]) are devoted to the study of spectral properties of systems of differential equations on the real line with periodic coefficients. Presently, interest in this subject is provoked by numerous applications to quantum mechanics and, in particular, to the study of conductivity of nanotubes (for example, see Korotyaev and Lobanov [18]), to optics, acoustics, and so on (see [34]). Note also that the case of difference periodic systems was considered by Korotyaev and Kutsenko [16, 17. Results that are directly relevant to the present paper will be discussed in more detail below.

Korotyev and Chelkak [5] constructed the Riemann surface of the Lyapunov function and found the asymptotic behavior of the 2-periodic (i.e., periodic and antiperiodic) spectrum and of the branch points of the Lyapunov function (resonances) for the Schrödinger operator with a periodic real $(N \times N)$-matrix potential (for $N=2$, see [2]). Moreover, in that paper, a conformal mapping related to the integral state density was constructed and studied; the lengths of gaps were estimated in terms of the norm of the potential, and some new trace formulas were derived. The case of a matrix complex potential and first-order systems was considered by Korotyaev in [13, 15]. We note the following results for the Schrödinger operator with a periodic $(N \times N)$-matrix potential and for the $(2 N \times 2 N)$-matrix operator of the first order.

1) Systems of differential equations may split into independent scalar equations. In this case, $\rho=0$.

2 ) In the case of the Schrödinger operator, the 2-periodic eigenvalues and resonances go to $+\infty$; in the case of the first-order operator, the 2-periodic eigenvalues and resonances go to $\pm \infty$.

3) Roughly speaking, the spectrum at high energy has maximal multiplicity $2 N$. 
4) There is a class of potentials for which there are infinitely many gaps in the spectrum of the corresponding operator. At the same time, under certain conditions on the potential, the number of gaps in the spectrum is finite (see also [23]).

Papanicolaou [26, 27] and 28] (jointly with Kravvaritis) studied spectral properties of the Euler-Bernoulli equation $\left(a y^{\prime \prime}\right)^{\prime \prime}=\lambda b y$ with periodic coefficients $a, b>0$. He showed that the spectrum lies in the interval $[0, \infty)$ and consists of bands separated by gaps. The endpoints of gaps are periodic or antiperiodic eigenvalues. Any point of the spectrum has multiplicity two. The beginning of the spectrum coincides with the point 0 , which is a periodic eigenvalue and simultaneously a simple resonance. All the remaining resonances are negative and have multiplicity 1 or 2 . It was proved that the spectrum of the Dirichlet problem lies in gaps and some results (Borg-type theorems) related to the inverse spectral problem were obtained.

In [1, the authors of the present paper arrived at the following results for the operator $H=\frac{d^{4}}{d t^{4}}+q$ :

1) the existence of real and nonreal branch points of the Lyapunov function for potentials of a certain kind; 2) the asymptotic behavior of 2-periodic eigenvalues and resonances in terms of the Fourier coefficients of the function $q$; 3) if $q \rightarrow 0$, then at the beginning of the spectrum there is a small band of multiplicity 4, and its asymptotic behavior was found; the other spectrum has multiplicity 2.

An explicit formula for the spectral expansion of the general periodic operator of order $2 N$ was obtained by Tkachenko in [33] (for the case of $N=2$, see [31, 32]). Mikhailets and Molyboga 21, 22, obtained asymptotic estimates for the periodic and antiperiodic eigenvalues of the operator $(-1)^{N} \frac{d^{2 N}}{d t^{2 N}}+q$, where $q$ is a periodic distribution.

In the present paper, we extend the results of [1] to the case of the general periodic fourth-order operator. Moreover, we introduce a natural numeration of spectral bands. Namely, we show that precisely one spectral band $\sigma_{n}$ corresponds to each pair $\lambda_{n-1}^{+}, \lambda_{n}^{-}$, $n \geq 1$. For every band $\sigma_{n}$, two cases may occur: 1 ) the endpoints of the band are the points $\lambda_{n-1}^{+}$and $\lambda_{n}^{-} ; 2$ ) one of the endpoints of the band is the point $\lambda_{n-1}^{+}$or $\lambda_{n}^{-}$and the other endpoint is a resonance. The asymptotic behavior of the spectral bands at high energy is found.

We denote by $\mathcal{R}$ the set of all real resonances (the zeros of the function $\rho$ ). In Lemma 5.1 we show that if $\Delta_{1}(r)=\Delta_{2}(r) \in(-1,1)$ for some $r \in \mathcal{R}$, then $r$ is a resonance of multiplicity 1 or 2 . If $r$ has multiplicity 2 , then the functions $\Delta_{1}$ and $\Delta_{2}$ are analytic in a neighborhood of $r$.

Definition 1. The set $\mathcal{R}_{0} \subset \mathcal{R}$ consists of resonances $r$ such that $\Delta_{1}(r)=\Delta_{2}(r) \in$ $(-1,1)$ and one of the following two conditions is satisfied:

a) $r$ is a simple resonance,

b) $r$ has multiplicity 2 and $\Delta_{1}^{\prime}(r) \Delta_{2}^{\prime}(r)<0$.

The asymptotic formulas (1.6) imply that the number of elements of the set $\mathcal{R}_{0}$ is finite.

In the next theorem we show that precisely one natural nondegenerate band $\sigma_{n}$ corresponds to every pair $\left\{\lambda_{n}^{-}, \lambda_{n-1}^{+}\right\}, n \geq 1$. Inside every interval $\sigma_{n}$, there are finitely many $(\geq 0)$ resonances, each not belonging to $\mathcal{R}_{0}$ and having multiplicity 2 . Consequently, the functions $\Delta_{1}$ and $\Delta_{2}$ are real-analytic on each of the intervals $\sigma_{n}$. We define functions $\Delta_{ \pm}$by the relations

$$
\Delta_{+}(\lambda)=\max \left\{\Delta_{1}(\lambda), \Delta_{2}(\lambda)\right\}, \quad \Delta_{-}(\lambda)=\min \left\{\Delta_{1}(\lambda), \Delta_{2}(\lambda)\right\}
$$

on the set $\{\lambda \in \mathbb{R}: \rho(\lambda)>0\}$. Three cases are possible (see Figure 1). 

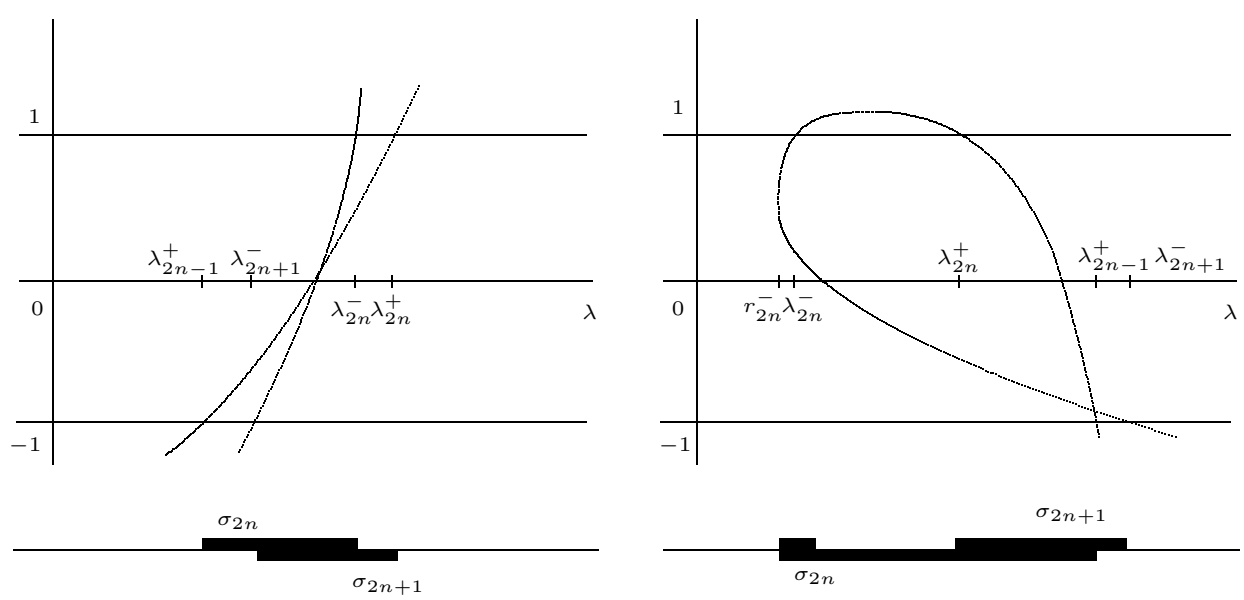

FiguRE 1. Spectral bands: case I is shown on the left; case II is shown on the right.

Case I. The band $\sigma_{n}$ has the form $\sigma_{n}=\left[\lambda_{n-1}^{+}, \lambda_{n}^{-}\right]$or $\sigma_{n}=\left[\lambda_{n}^{-}, \lambda_{n-1}^{+}\right]$, where the points $\lambda_{n}^{-}$and $\lambda_{n-1}^{+}$are zeros of the function $\Delta_{\nu}^{2}-1$ for a certain $\nu=+,-$; moreover, $\Delta_{\nu}$ is continuous and monotone on the interval $\sigma_{n}$ and $\Delta_{\nu}\left(\sigma_{n}\right)=[-1,1]$.

Note that, in case I, $\lambda_{n-1}^{+} \neq \lambda_{n}^{-}$and the points $\lambda_{n}^{-}$and $\lambda_{n-1}^{+}$are the endpoints of the band $\sigma_{n}$.

Cases II, III. The band $\sigma_{n}$ has the form $\sigma_{n}=\sigma_{n}^{-} \cup \sigma_{n}^{+}$, where

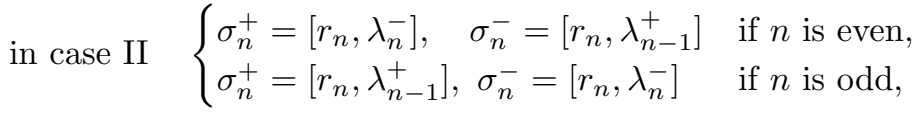

$$
\begin{aligned}
& \text { in case III }\left\{\begin{array}{lll}
\sigma_{n}^{+}=\left[\lambda_{n}^{-}, r_{n}\right], & \sigma_{n}^{-}=\left[\lambda_{n-1}^{+}, r_{n}\right] & \text { if } n \text { is even, } \\
\sigma_{n}^{+}=\left[\lambda_{n-1}^{+}, r_{n}\right], & \sigma_{n}^{-}=\left[\lambda_{n}^{-}, r_{n}\right] & \text { if } n \text { is odd, }
\end{array}\right.
\end{aligned}
$$

and $r_{n}$ is a resonance lying in the set $\mathcal{R}_{0}$. The functions $\Delta_{ \pm}$are continuous and monotone on the segments $\sigma_{n}^{ \pm}$, respectively, and the relations

$$
\Delta_{+}\left(\sigma_{n}^{+}\right)=\left[\beta_{n}, 1\right], \Delta_{-}\left(\sigma_{n}^{-}\right)=\left[-1, \beta_{n}\right], \text { where } \beta_{n}=\Delta_{+}\left(r_{n}\right)=\Delta_{-}\left(r_{n}\right) \in(-1,1)
$$

are valid. There are no resonances inside the interval $\sigma_{n}^{+} \cap \sigma_{n}^{-}$.

In cases II and III, it may happen that $\lambda_{n-1}^{+}=\lambda_{n}^{-}$. The points $\lambda_{n}^{-}$and $\lambda_{n-1}^{+}$belong to the band $\sigma_{n}$, but only one of them is the endpoint of the band and the other endpoint is the resonance $r_{n}$ from the set $\mathcal{R}_{0}$. The resonance is the left endpoint in case II and the right endpoint in case III.

Theorem 1.2. i) $\sigma(H)=\bigcup_{n>1} \sigma_{n}$, where the $\sigma_{n} \subset \mathbb{R}$ are nondegenerate finite intervals. For every $\sigma_{n}, n \geq 1$, precisely one of the cases I, II, or III is realized; moreover, case III is impossible for $\sigma_{1}$. The resonances inside every interval $\sigma_{n}$ have multiplicity 2 and do not belong to $\mathcal{R}_{0}$. Next,

$$
\sigma_{n} \cap \sigma_{n+2}=\varnothing, \quad n \geq 1 .
$$

ii) Let $r$ be a resonance lying inside a band $\sigma_{n}$ with some $n \geq 1$, and let $\tau_{1}(r)=\tau_{2}(r) \in$ $\mathbb{C}_{+}$. Then $\tau_{1}$ and $\tau_{2}$ are analytic in a neighborhood of $r$, and the following relations are 
valid:

$$
\left|\tau_{j}(\lambda)\right|=1, \quad i \tau_{j}^{-1}(r) \tau_{j}^{\prime}(r)>0(\text { or }<0) \quad \text { for both values of } j=1,2 .
$$

iii) Every resonance of the set $\mathcal{R}_{0}$ is an endpoint of the band $\sigma_{n}$ for some $n \geq 1$.

iv) The spectrum $\sigma(H)$ has multiplicity 4 on the set

$$
\mathfrak{S}_{4}=\left(\bigcup_{n \geq 1}\left(\sigma_{n} \cap \sigma_{n+1}\right)\right) \cup\left(\bigcup_{n: \text { II or III is valid }}\left(\sigma_{n}^{-} \cap \sigma_{n}^{+}\right)\right)
$$

and multiplicity 2 on the set $\mathfrak{S}_{2}=\sigma(H) \backslash \mathfrak{S}_{4}$.

Remarks. 1) For $q=0$ and small $p$, we have case II for $\sigma_{1}$ and case I for all $\sigma_{n}, n \geq 2$ (see Theorem 1.4 and Figure 3). The graph of the Lyapunov function $\Delta$ for arbitrary coefficients $p$ and $q$ may have a rather complicated form (see Figure 2, which depicts case III for $\sigma_{4}$ and case I for the remaining $\sigma_{n}, n \geq 1$ ). Nevertheless, for the operator $H$ with arbitrary $p$ and $q$, only case I is realized for all $\sigma_{n}$ and $n$ sufficiently large (see Theorem 1.3). For the Hill operator, only case I is always realized.

2 ) The first band in the spectrum of the operator $\left(-\frac{d^{2}}{d t^{2}}+p\right)^{2}$ with constant $p=-\pi^{2}-\delta$ and any sufficiently small $\delta>0$ (for example, with $\delta=1$ ) satisfies condition I and contains one resonance.

3) The example of the operator $\left(-\frac{d^{2}}{d t^{2}}-p_{0}+p_{1}\right)^{2}$ with a large constant $p_{0}>0$ and a small periodic $p_{1}$ shows that the bands $\sigma_{n}$ with neighboring numbers may overlap, i.e., $\sigma_{n} \cap \sigma_{n+1} \neq \varnothing$ for some $n$.

4) The assumption $\tau_{1}(r)=\tau_{2}(r) \in \mathbb{C}_{+}$in part ii) of the theorem does not reduce generality. Indeed, since $r$ lies inside a band, all the multipliers $\tau_{1}, \tau_{1}^{-1}, \tau_{2}, \tau_{2}^{-1}$ are equal to one by modulus and are not real. For this reason, we may assume that $\tau_{1}$ and $\tau_{2}$ lie in the upper half-plane and $\tau_{1}^{-1}$ and $\tau_{2}^{-1}$ are placed in the lower half-plane. Relations (1.13) mean that, as $\lambda$ increases in the interval $(r-\delta, r+\delta)$ with some $\delta>0$, the two multipliers $\tau_{1}$ and $\tau_{2}$ move along the unit circle in the same direction; for $\lambda=r$, one of them outruns the other. For periodic systems of linear differential equations, a result similar to Theorem 1.2 , ii) was obtained by Krĕn [19.

Denote by $r_{0}^{-}, r_{n}^{ \pm}, n \geq 1$, the sequence of resonances in $\mathbb{C}$ enumerated (counting multiplicities) in such a way that $\left|r_{0}^{-}\right| \leq\left|r_{1}^{+}\right| \leq\left|r_{1}^{-}\right| \leq\left|r_{2}^{+}\right| \leq \cdots$. We state a theorem on the asymptotic behavior of 2-periodic eigenvalues and resonances at high energy and on the restoration of the spectrum of $H$.

Theorem 1.3. i) For all $n$ beginning with some $n_{0} \geq 1$, the spectral bands $\sigma_{n}$ have the form $\sigma_{n}=\left[\lambda_{n-1}^{+}, \lambda_{n}^{-}\right]$and $\lambda_{n_{0}}^{-} \leq \lambda_{n_{0}}^{+}<\lambda_{n_{0}+1}^{-} \leq \lambda_{n_{0}+1}^{+}<\cdots$. The spectrum of $H$ in $\sigma_{n}$, $n \geq n_{0}$, has multiplicity 2. Moreover, if $\lambda_{n}^{-}<\lambda_{n}^{+}$for some $n \geq n_{0}$, then the interval $\left(\lambda_{n}^{-}, \lambda_{n}^{+}\right)$is a gap. We have the following asymptotic relations:

$$
\begin{aligned}
& r_{n}^{ \pm}=-4(\pi n)^{4}+2 \widehat{p}_{0}(\pi n)^{2} \pm \sqrt{2} \pi n\left|\widehat{p_{n}^{\prime}}\right|+O(1), \\
& \lambda_{n}^{ \pm}=(\pi n)^{4}-\widehat{p}_{0}(\pi n)^{2} \pm \pi n \frac{\left|\widehat{p_{n}^{\prime}}\right|}{2}+O(1)
\end{aligned}
$$

as $n \rightarrow \infty$, where $\widehat{p_{n}}=\int_{0}^{1} p(t) e^{-i 2 \pi n t} d t, n \in \mathbb{Z}$.

In particular, if $\left|\widehat{p_{n}^{\prime}}\right| \geq n^{-\alpha}$ for all sufficiently large $n$ and some $\alpha, 0<\alpha<1$, then there exist infinitely many gaps $\gamma_{n} \neq \varnothing$, and $\left|\gamma_{n}\right| \rightarrow \infty$ as $n \rightarrow \infty$. Moreover, in this case, all the resonances except for a finite number of them are real and negative, and there exist infinitely many nonempty intervals $\left(r_{n}^{-}, r_{n}^{+}\right) \subset \mathbb{R}$, and $\left|r_{n}^{+}-r_{n}^{-}\right| \rightarrow \infty$ as $n \rightarrow \infty$. 


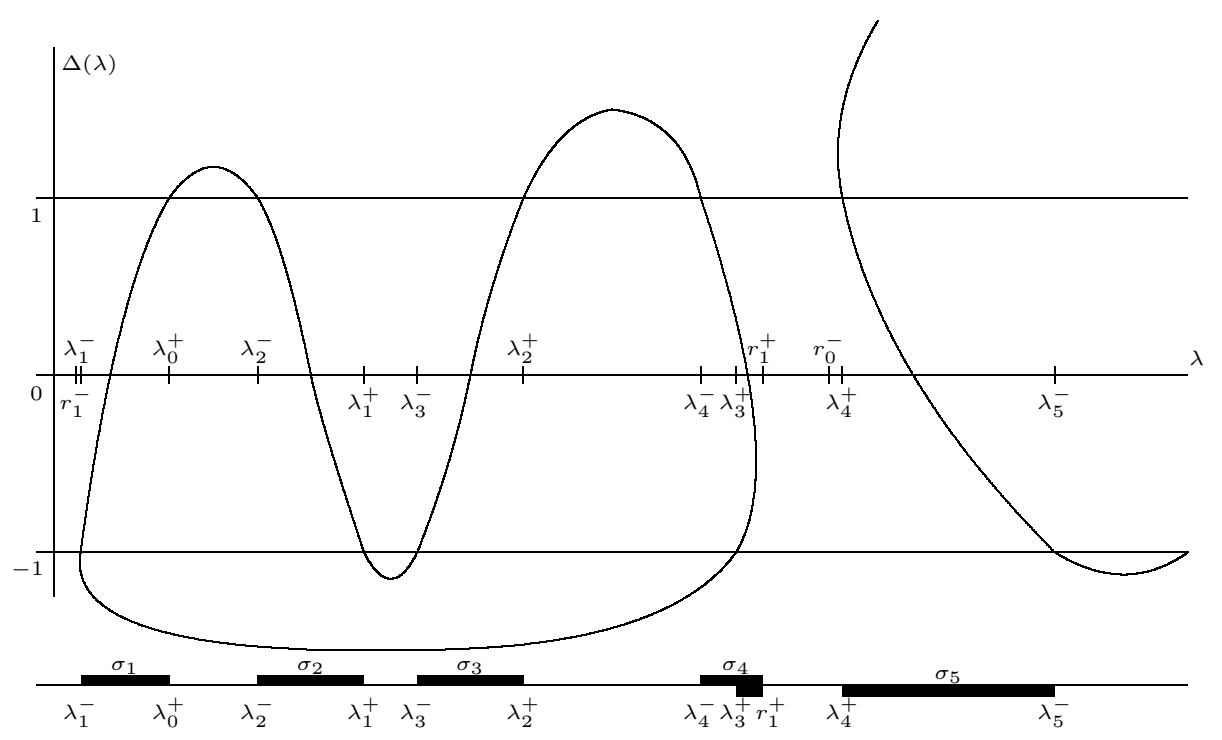

FiguRE 2. The graph of a possible function $\Delta$ and the spectrum $\sigma(H)$. As a numerical investigation shows, the graph of the Lyapunov function for the operator $H$ with $p=0, q=\eta \delta_{\text {per }}$ has a similar form; here $\delta_{\text {per }}$ is a 1-periodic delta-function and $\eta=7937.7$.

ii) The Lyapunov function and, thus, the spectrum of $H$ (counted with multiplicity), is uniquely determined by one of the following collections of numbers:

a) the 2-periodic spectrum;

b) the periodic (or antiperiodic) spectrum and the resonances.

Remarks. 1) By Lemma 3.3, the function $\rho$ has an odd number of zeros, counted with multiplicity, in any circle $|\lambda|<R$ with $R=4\left(\pi\left(N+\frac{1}{2}\right)\right)^{4}$ large. In particular, the Lyapunov function has at least one real branch point.

2) The 2-periodic eigenvalues of the operator $H$ go to $+\infty$, and the resonances go to $-\infty$.

3) For certain $p \neq 0$ and any $q \in L^{1}(0,1)$, the spectrum $\sigma(H)$ has infinitely many gaps $\gamma_{n}$, growing as $n \rightarrow \infty$. If $p=0$, then for any $q \in L^{1}(0,1)$, the gaps $\gamma_{n}$ decrease as $n \rightarrow \infty$ (see [1]).

In the following theorem, we show that the lowest gap in $\sigma(H)$ contains an interval of spectral multiplicity 4 for $q=0$ and $p \rightarrow 0$. In the sequel, sometimes we write $\rho(\lambda, p)$, $\Delta(\lambda, p, q), \ldots$ instead of $\rho(\lambda), \Delta(\lambda), \ldots$ when we want to emphasize the dependence of the corresponding function on the coefficients $p$ and $q$.

Theorem 1.4. Let $H_{\varepsilon}=\frac{d^{4}}{d t^{4}}+\varepsilon \frac{d}{d t} p \frac{d}{d t}, \varepsilon \in \mathbb{R}$, and suppose that $\int_{0}^{1} p(t) d t=0$. Then there exist two real-analytic functions $r_{0}^{-}(\varepsilon)$ and $\lambda_{0}^{+}(\varepsilon)$ in the circle $\left\{|\varepsilon|<\varepsilon_{1}\right\}$ with some $\varepsilon_{1}>0$ such that $r_{0}^{-}(\varepsilon)$ is a simple zero of the function $\rho(\cdot, \varepsilon p), \lambda_{0}^{+}(\varepsilon)$ is a simple zero of the function $D_{+}(\cdot, \varepsilon p)$, and $r_{0}^{-}(0)=\lambda_{0}^{+}(0)=0$. These functions admit the following asymptotic formulas:

$$
\begin{aligned}
& r_{0}^{-}(\varepsilon)=2 \varepsilon^{2}\left(4 v_{1}-v_{2}\right)+O\left(\varepsilon^{3}\right), \quad \lambda_{0}^{+}(\varepsilon)=2 \varepsilon^{2}\left(4 v_{1}-v_{2}\right)+O\left(\varepsilon^{3}\right), \\
& \lambda_{0}^{+}(\varepsilon)-r_{0}^{-}(\varepsilon)=4 A^{2} \varepsilon^{4}+O\left(\varepsilon^{5}\right), \quad A=\frac{v_{2}}{12}-\frac{4 v_{1}}{3}=\frac{1}{4} \sum_{n \neq 0} \frac{\left|\hat{p}_{n}\right|^{2}}{(2 \pi n)^{2}}>0,
\end{aligned}
$$




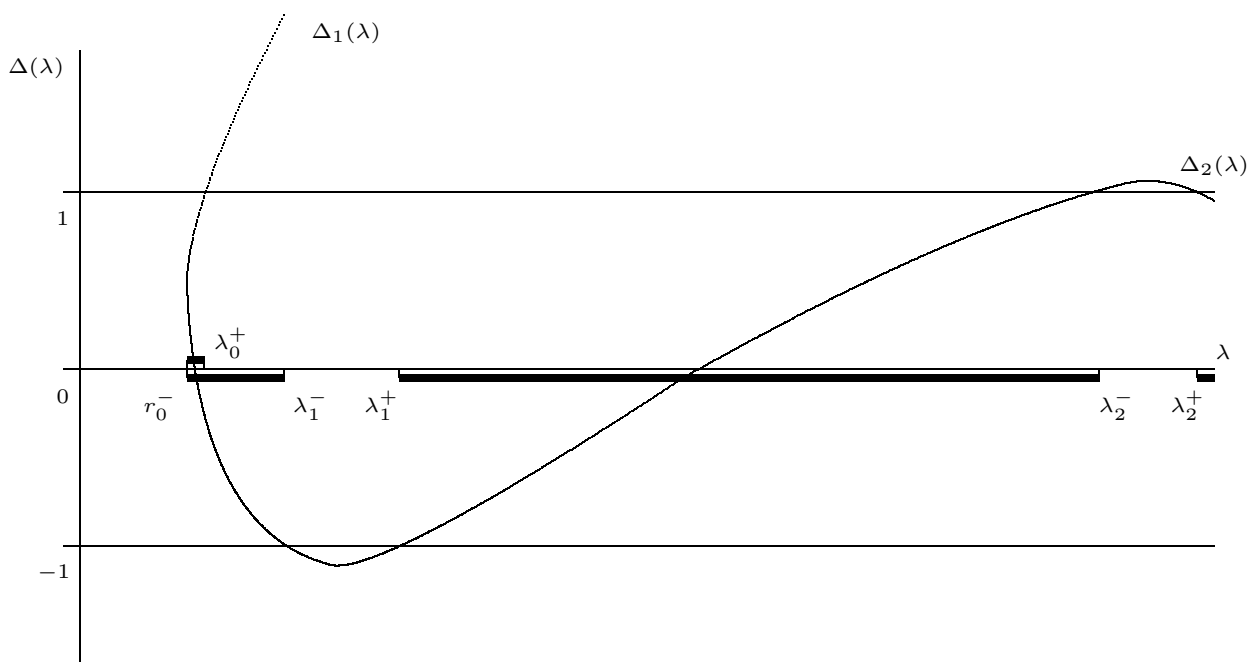

Figure 3. The spectrum of $H_{\varepsilon}$ for small $\varepsilon$.

as $\varepsilon \rightarrow 0$, where

$$
v_{\nu}=\frac{1}{4} \int_{0}^{\nu} d t \int_{0}^{t} p(s) p(t)(\nu-t+s)(t-s) d s, \quad \nu=1,2 .
$$

Moreover, if $\varepsilon \in\left(-\varepsilon_{1}, \varepsilon_{1}\right) \backslash\{0\}$, then $r_{0}^{-}(\varepsilon)<\lambda_{0}^{+}(\varepsilon)$ and the spectrum $\sigma\left(H_{\varepsilon}\right)$ in the interval $\left(r_{0}^{-}(\varepsilon), \lambda_{0}^{+}(\varepsilon)\right)$ has multiplicity 4 . The spectrum outside this interval has multiplicity 2.

Remark. 1) The graph of the Lyapunov function for $q=0$ and small $p$ is depicted in Figure 3 .

2) For constant $p<0$ and $q=0$, any point of the spectrum has multiplicity 2 (this follows from relations (2.19); see below at the end of $\S 2$ ). Therefore, the assumption $\int_{0}^{1} p(t) d t=0$ of Theorem [1.4 is essential. Note also that for constant $p>0$ and $q=0$, the spectrum in the interval $\left(-\frac{p^{2}}{4}, 0\right)$ has multiplicity 4 , and the spectrum outside this interval has multiplicity 2 .

3) The case of the operator $H$ with $p=0$ and $q \rightarrow 0$ was considered in [1, and the results are similar.

4) It is important to note that the result in the general case is more complicated. Namely, one can choose $p \neq 0, q \neq 0,(p, q) \rightarrow 0$ such that the spectrum $\sigma(H)$ has always multiplicity two. This follows from a result due to Papanicolaou in 26, where it was shown that any point of the spectrum of the Euler-Bernoulli equation $\left(a y^{\prime \prime}\right)^{\prime \prime}=\lambda b y$ with any periodic $a>0$ and $b>0$ has multiplicity 2 .

In conclusion, we mention that the operators $H=\frac{d^{4}}{d x^{4}}+\frac{d}{d x} p \frac{d}{d x}+q$ and $K=8 \frac{d^{3}}{d x^{3}}+$ $6 p \frac{d}{d x}+3 p^{\prime}$ form a Lax pair, where $p=p(x, t), q=q(x, t), x$ is a space coordinate, and $t$ is time (for example, see the survey [9]). The corresponding nonlinear equation $\partial_{t} H=[H, K]$ was written out explicitly in [11] and has the form

$$
\left\{\begin{array}{l}
\partial_{t} p=10 \partial_{x}^{3} p+6 p \partial_{x} p-24 \partial_{x} q \\
\partial_{t} q=3\left(\partial_{x}^{5} p+p \partial_{x}^{3} p+\partial_{x} p \partial_{x}^{2} p\right)-8 \partial_{x}^{3} q-6 p \partial_{x} q .
\end{array}\right.
$$

Now we describe the plan of our paper. In $\S 2$, we obtain the main properties of the fundamental solutions $\varphi_{j}, j \in \mathbb{N}_{3}$. In $\S 3$, we introduce and study solutions of equation (1.1) of the Jost type, which have good asymptotic behavior as $|\lambda| \rightarrow \infty$. The idea of using such solutions for finding the spectral asymptotic behavior of boundary value 
problems for higher-order equations is due to Birkhoff (see [25]). The results obtained enable one to find the asymptotic behavior of the monodromy matrix and of multipliers at high energy. Pertinent technical proofs are given in the Appendix. Moreover, in $\S 3$ we study the main properties of the Lyapunov function and the function $\rho$. In $\S 4$, Floquet solutions are investigated. In $\S 5$, we prove Theorems 1.2 1.3. In $\S 6$, we consider the operator $H$ with small coefficients and prove Theorem 1.4 .

\section{§2. Properties of fundamental solutions}

The fundamental solutions $\varphi_{j}^{0}(t, \lambda),(j, t, \lambda) \in \mathbb{N}_{3} \times \mathbb{R} \times \mathbb{C}$ of the nonperturbed equation $y^{\text {(iv) }}=\lambda y$ have the form

$$
\begin{array}{ll}
\varphi_{0}^{0}=\frac{\cosh z t+\cos z t}{2}, & \varphi_{1}^{0}=\frac{\sinh z t+\sin z t}{2 z}, \\
\varphi_{2}^{0}=\frac{\cosh z t-\cos z t}{2 z^{2}}, & \varphi_{3}^{0}=\frac{\sinh z t-\sin z t}{2 z^{3}} ;
\end{array}
$$

recall that $z=x+i y=\lambda^{1 / 4}, \arg z \in\left(-\frac{\pi}{4}, \frac{\pi}{4}\right]$. For a 1-periodic function $f \in L^{1}(0,1)$ we introduce the norm $\|f\|=\int_{0}^{1}|f(t)| d t<\infty$.

Lemma 2.1. i) Every function $\varphi_{j}(t, \cdot), j \in \mathbb{N}_{3}, t \in \mathbb{R}_{+}$, is an entire function real on $\mathbb{R}$ and

$$
\begin{aligned}
\max _{j, k \in \mathbb{N}_{3}}\left\{\left|\lambda^{\frac{j-k}{4}}\left(\varphi_{j}^{(k)}(m, \lambda)-\sum_{n=0}^{N-1} \varphi_{n, j}^{(k)}(m, \lambda)\right)\right|\right\} & \leq \frac{(\varkappa m)^{N}}{N !|z|_{1}^{N}} e^{(x+\varkappa) m}, \\
\varkappa & =\|p\|+\left\|p^{\prime}\right\|+\|q\|,
\end{aligned}
$$

for all $m \in \mathbb{N}, N \geq 0, \lambda \in \mathbb{C}$, where $|z|_{1}=\max \{1,|z|\}$. Moreover, the functions $T_{\nu}=\frac{1}{4} \operatorname{Tr} M^{\nu}, \nu=1,2$, are entire, real on $\mathbb{R}$, and obey the estimates

$$
\left|T_{\nu}(\lambda)\right| \leq e^{(x+\varkappa) \nu}, \quad\left|T_{\nu}(\lambda)-T_{\nu}^{0}(\lambda)\right| \leq \frac{\nu \varkappa}{|z|_{1}} e^{(x+\varkappa) \nu}, \quad \lambda \in \mathbb{C} .
$$

ii) Let $q=0$, and let $\hat{p}_{0}=p(0)=0$. Then

$$
\left|T_{\nu}(\lambda)-T_{\nu}^{0}(\lambda)-\eta_{\nu}(\lambda)\right| \leq \frac{(\nu \varkappa)^{3}}{6|z|_{1}^{3}} e^{(x+\varkappa) \nu}, \quad \nu=1,2, \quad \lambda \in \mathbb{C},
$$

where

$$
\eta_{\nu}(\lambda)=\frac{1}{4} \int_{0}^{\nu} d t \int_{0}^{t} p(s) p(t) \varphi_{1}^{0}(\nu-t+s, \lambda) \varphi_{1}^{0}(t-s, \lambda) d s .
$$

Proof. For any fixed $(j, t) \in \mathbb{N}_{3} \times \mathbb{R}_{+}$, the functions $\varphi_{j}^{0}(t, \cdot)$ are entire, and we have

$$
\sum_{m=0}^{3}\left(\varphi_{j}^{0}\right)^{(m)}(t)\left(\varphi_{m}^{0}\right)^{(k)}(s)=\varphi_{j-k}^{0}(t+s), \quad\left|\varphi_{j}^{0}(t)\right| \leq \frac{e^{x t}+e^{|y| t}}{2|z|_{1}^{j}} \leq \frac{e^{x t}}{|z|_{1}^{j}},
$$

where $(k, s) \in \mathbb{N}_{3} \times \mathbb{R}_{+}, k \leq j$. Throughout the proof, $\varphi_{j}^{0}(t)=\varphi_{j}^{0}(t, \lambda), \ldots$

i) The fundamental solutions $\varphi_{j}$ satisfy the equation

$$
\varphi_{j}(t)=\varphi_{j}^{0}(t)-\int_{0}^{t} \varphi_{3}^{0}(t-s) u_{j}(s) d s, \quad u_{j}=\left(p \varphi_{j}^{\prime}\right)^{\prime}+q \varphi_{j}, \quad(j, t) \in \mathbb{N}_{3} \times \mathbb{R}_{+} .
$$

Consequently, the functions $u_{j}$ satisfy the integral equation

$$
\begin{aligned}
u_{j}(t) & =u_{0, j}(t)-\int_{0}^{t} K_{3}(t, t-s) u_{j}(s) d s, \quad \text { where } \quad u_{0, j}(t)=K_{j}(t, t), \\
K_{j}(t, s) & =p(t)\left(\varphi_{j}^{0}\right)^{\prime \prime}(s)+p^{\prime}(t)\left(\varphi_{j}^{0}\right)^{\prime}(s)+q(t) \varphi_{j}^{0}(s) .
\end{aligned}
$$


Iterations in (2.8) yield

$$
u_{j}(t)=\sum_{n \geq 0} u_{n, j}(t), \quad u_{n+1, j}(t)=-\int_{0}^{t} K_{3}(t, t-s) u_{n, j}(s) d s,
$$

whence

$$
u_{n, j}(t)=(-1)^{n} \int_{\substack{0<t_{1}<t_{2}<\cdots \\<t_{n}<t_{n+1}=t}}\left(\prod_{k=1}^{n} K_{3}\left(t_{k+1}, t_{k+1}-t_{k}\right)\right) u_{0, j}\left(t_{1}\right) d t_{1} d t_{2} \cdots d t_{n} .
$$

Substituting (2.10) in (2.7), we obtain

$$
\varphi_{j}(t)=\sum_{n \geq 0} \varphi_{n, j}(t), \quad \varphi_{n, j}(t)=-\int_{0}^{t} \varphi_{3}^{0}(t-s) u_{n-1, j}(s) d s, \quad \varphi_{0, j}=\varphi_{j}^{0} .
$$

Substituting (2.11) in the last formulas, we get

$$
\begin{aligned}
\varphi_{n, j}(t)=( & -1)^{n} \int_{\substack{0<t_{1}<t_{2}<\cdots \\
<t_{n}<t}} \varphi_{3}^{0}\left(t-t_{n}\right) \\
& \times\left(\prod_{k=1}^{n-1} K_{3}\left(t_{k+1}, t_{k+1}-t_{k}\right)\right) u_{0, j}\left(t_{1}\right) d t_{1} d t_{2} \cdots d t_{n} .
\end{aligned}
$$

From (2.9) we have $\left|K_{j}(t, s)\right| \leq|z|_{1}^{2-j} e^{x s}\left(|p(t)|+\left|p^{\prime}(t)\right|+|q(t)|\right)$, where we have used the inequalities (2.6). Substituting these estimates in (2.13), we obtain

$$
\left|\varphi_{n, j}(t)\right| \leq \frac{e^{x t}}{|z|_{1}^{j+n} n !}\left(\int_{0}^{t}\left(|p(s)|+\left|p^{\prime}(s)\right|+|q(s)|\right) d s\right)^{n} .
$$

These estimates show that for any fixed $t \in \mathbb{R}_{+}$, the formal series (2.12) converges absolutely and uniformly on bounded subsets in $\mathbb{C}$. Each summand of this series is an entire function real for $\lambda \in \mathbb{R}$. Therefore, the sum is also an entire function real for $\lambda \in \mathbb{R}$. Summing the majorants, we obtain estimates (2.2).

Now we prove (2.3). For $\nu=1,2$, the relations

$$
T_{\nu}=\frac{1}{4} \operatorname{Tr} M^{\nu}=\frac{1}{4} \operatorname{Tr} M(\nu)=\frac{1}{4} \sum_{j=0}^{3} \varphi_{j}^{(j)}(\nu)=\sum_{n \geq 0} f_{\nu n}, \quad f_{\nu n}=\frac{1}{4} \sum_{j=0}^{3} \varphi_{n, j}^{(j)}(\nu),
$$

are valid; here we have used (2.12). From (2.13), we get

$$
\begin{aligned}
\varphi_{n, j}^{(j)}(t)=(-1)^{n} & \int_{\substack{0<t_{1}<t_{2}<\cdots \\
<t_{n}<t}}\left(\varphi_{3}^{0}\right)^{(j)}\left(t-t_{n}\right) \\
& \times\left(\prod_{k=1}^{n-1} K_{3}\left(t_{k+1}, t_{k+1}-t_{k}\right)\right) u_{0, j}\left(t_{1}\right) d t_{1} d t_{2} \cdots d t_{n} .
\end{aligned}
$$

Then inequalities (2.6) yield $\left|\varphi_{n, j}^{(j)}(\nu, \lambda)\right| \leq \frac{(\nu \varkappa)^{n}}{n !|z|_{1}^{n}} e^{x \nu}$, whence we have

$$
\left|f_{\nu n}(\lambda)\right| \leq \frac{(\nu \varkappa)^{n}}{n !|z|_{1}^{n}} e^{x \nu}, \quad n \geq 0 .
$$

This inequality shows that the series (2.15) converges absolutely and uniformly on bounded subsets in $\mathbb{C}$. Each summand of this series is an entire function real on $\mathbb{R}$. Consequently, the sum equal to $T_{\nu}$ is also an entire function real on $\mathbb{R}$. Summing the majorants and taking the relation $f_{\nu 0}=T_{\nu}^{0}$ into account, we obtain (2.3). 
From (2.15) and (2.16) it follows that

$$
\left|T_{\nu}-T_{\nu}^{0}-f_{\nu 1}-f_{\nu 2}\right| \leq \frac{(\nu \varkappa)^{3}}{6|z|_{1}^{3}} e^{(x+\varkappa) \nu}
$$

Assume that

$$
f_{\nu 1}=0, \quad f_{\nu 2}=\eta_{\nu} .
$$

Next, substituting (2.18) in (2.17), we get (2.4).

Now we prove (2.18). Substituting the last relation in (2.8), using (2.12), and taking $q=0$ into account, we arrive at the formula

$$
\varphi_{1, k}^{(k)}(\nu)=-\int_{0}^{\nu}\left(\varphi_{3}^{0}\right)^{(k)}(\nu-t)\left(p(t)\left(\varphi_{k}^{0}\right)^{\prime \prime}(t)+p^{\prime}(t)\left(\varphi_{k}^{0}\right)^{\prime}(t)\right) d t
$$

Substituting this in (2.15) and using the first relation in (2.6), we get

$$
4 f_{\nu 1}=-\varphi_{1}^{0}(\nu) \int_{0}^{\nu} p(t) d t-\varphi_{2}^{0}(\nu) \int_{0}^{\nu} p^{\prime}(t) d t
$$

since $\hat{p}_{0}=0$, this provides the first relation in (2.18). Similar calculations that involve (2.6) -(2.12) and (2.15) lead to the relations

$$
\begin{gathered}
4 f_{\nu 2}=\sum_{k=0}^{3} \int_{0}^{\nu} \int_{0}^{t} K_{3}(t, t-s)\left(\varphi_{3}^{0}\right)^{(k)}(\nu-t)\left(p(s)\left(\varphi_{k}^{0}\right)^{\prime \prime}(s)+p^{\prime}(s)\left(\varphi_{k}^{0}\right)^{\prime}(s)\right) d s d t \\
=\int_{0}^{\nu} \int_{0}^{t}\left(p(t) \varphi_{1}^{0}(t-s)+p^{\prime}(t) \varphi_{2}^{0}(t-s)\right)\left(p(s) \varphi_{1}^{0}(\nu-t+s)\right. \\
\left.+p^{\prime}(s) \varphi_{2}^{0}(\nu-t+s)\right) d s d t \\
=\int_{0}^{\nu} \int_{0}^{t}\left(p(t) p(s) \widetilde{\varphi}_{11}(t-s)+p^{\prime}(t) p(s) \widetilde{\varphi}_{12}(t-s)\right. \\
\left.\quad+p(t) p^{\prime}(s) \tilde{\varphi}_{21}(t-s)+p^{\prime}(t) p^{\prime}(s) \tilde{\varphi}_{22}(t-s)\right) d s d t
\end{gathered}
$$

where $\widetilde{\varphi}_{k m}(t)=\varphi_{k}^{0}(\nu-t) \varphi_{m}^{0}(t)$. Since $p(0)=0$, integration by parts yields

$$
\int_{0}^{\nu} \int_{0}^{t} p^{\prime}(t) p^{\prime}(s) \widetilde{\varphi}_{22}(t-s) d s d t=-\int_{0}^{\nu} \int_{0}^{t} p^{\prime}(t) p(s)\left(\widetilde{\varphi}_{12}(t-s)-\widetilde{\varphi}_{21}(t-s)\right) d s d t .
$$

Then $4 f_{\nu 2}=\int_{0}^{\nu} \int_{0}^{t} p(t) p(s) \widetilde{\varphi}_{11}(t-s) d s d t+J$, where

$$
\begin{aligned}
J= & \int_{0}^{\nu} \int_{0}^{t}\left(p(t) p^{\prime}(s)+p^{\prime}(t) p(s)\right) \tilde{\varphi}_{21}(t-s) d s d t \\
= & \int_{0}^{\nu} p(t) \int_{0}^{t} p^{\prime}(s) \widetilde{\varphi}_{21}(t-s) d s d t+\int_{0}^{\nu} p(s) \int_{s}^{\nu} p^{\prime}(t) \widetilde{\varphi}_{21}(t-s) d t d s \\
= & \int_{0}^{\nu} p(t) \int_{0}^{t} p(s)\left(\widetilde{\varphi}_{20}(t-s)-\widetilde{\varphi}_{11}(t-s)\right) d s d t \\
& \quad-\int_{0}^{\nu} p(s) \int_{s}^{\nu} p(t)\left(\widetilde{\varphi}_{20}(t-s)-\widetilde{\varphi}_{11}(t-s)\right) d t d s=0 .
\end{aligned}
$$

We have $4 f_{\nu 2}=\int_{0}^{\nu} d t \int_{0}^{t} p(t) p(s) \widetilde{\varphi}_{11}(t-s) d s$, which provides the second relation in (2.18). 
Note that if the coefficients $p$ and $q$ are constant, then

$$
\begin{aligned}
T_{\nu} & =\frac{\cosh \nu z_{-}+\cos \nu z_{+}}{2}, \quad \rho=\frac{\left(\cosh z_{-}-\cos z_{+}\right)^{2}}{4}, \\
D_{ \pm} & =\left(\cos z_{+} \mp 1\right)\left(\cosh z_{-} \mp 1\right), \quad \Delta_{1}=\cosh z_{-}, \quad \Delta_{2}=\cos z_{+}, \\
z_{ \pm} & =\sqrt{\sqrt{\lambda+\frac{p^{2}}{4}-q} \pm \frac{p}{2}}, \quad p, q=\mathrm{const}, \quad \sqrt{1}=1 .
\end{aligned}
$$

\section{§3. Asymptotic Behavior at high Energy}

To investigate the asymptotic behavior of the monodromy matrix for large $|\lambda|$, we apply the standard Birkhoff approach, which involves fundamental solutions such as Jost solutions (see [25]). We introduce a diagonal matrix $\Omega(\lambda)=\left(\delta_{k j} \omega_{j}(\lambda)\right)_{k, j=0}^{3}$, which takes a constant value in each of the half-planes $\mathbb{C}_{ \pm}$and is given by the formulas

$$
\begin{aligned}
\omega_{0}(\lambda) & =-\omega_{3}(\lambda)=1, \quad \omega_{2}(\lambda)=-\omega_{1}(\lambda)=i, \quad \operatorname{Im} \lambda \geq 0, \\
\Omega(\lambda) & =\bar{\Omega}(\bar{\lambda}), \quad \operatorname{Im} \lambda<0 .
\end{aligned}
$$

From (3.1) it follows that

$$
\begin{aligned}
\operatorname{Re}\left(z \omega_{0}\right) & \geq \operatorname{Re}\left(z \omega_{1}\right) \geq \operatorname{Re}\left(z \omega_{2}\right) \geq \operatorname{Re}\left(z \omega_{3}\right), \quad z=\lambda^{1 / 4}, \\
\arg z & \in\left(-\frac{\pi}{4}, \frac{\pi}{4}\right], \quad \lambda \in \mathbb{C}, \omega_{j}=\omega_{j}(\lambda) .
\end{aligned}
$$

We introduce solutions $\vartheta_{j}(t, \lambda),(j, t, \lambda) \in \mathbb{N}_{3} \times[0,1] \times \mathbb{C}$, of the type of Jost solutions, which satisfy equation (1.11) and the asymptotic formula $\vartheta_{j}(t, \lambda)=e^{z \omega_{j} t}(1+o(1))$ as $|\lambda| \rightarrow \infty$ for each fixed $t \in[0,1]$. More precisely, we choose $\vartheta_{j}(t, \lambda)$ satisfying the integral equations

$$
\begin{aligned}
\vartheta_{j}(t, \lambda)=e^{z \omega_{j} t} & +\frac{1}{4 z^{3}} \int_{t}^{1} \sum_{n=0}^{j-1} \omega_{n} e^{z \omega_{n}(t-s)} g_{j}(s, \lambda) d s \\
& -\frac{1}{4 z^{3}} \int_{0}^{t} \sum_{n=j}^{3} \omega_{n} e^{z \omega_{n}(t-s)} g_{j}(s, \lambda) d s,
\end{aligned}
$$

where $g_{j}=\left(p \vartheta_{j}^{\prime}\right)^{\prime}+q \vartheta_{j}$. Each of these equations has a unique solution for all $|\lambda|$ sufficiently large, and this solution satisfies (1.1) (see [25]). Let

$$
\Lambda_{r}=\left\{\lambda \in \mathbb{C}:|\lambda|>r^{4} \max \left\{1, \varkappa^{4}\right\}\right\}, \quad r>0, \quad \Lambda_{r}^{ \pm}=\Lambda_{r} \cap \mathbb{C}^{ \pm} ;
$$

we recall that $\varkappa=\|p\|+\left\|p^{\prime}\right\|+\|q\|$. The proof of the following lemma is fully technical and will be given in the Appendix.

Lemma 3.1. i) We have

$$
\begin{aligned}
M & =\Theta_{0} \Phi e^{z \Omega} \Theta_{0}^{-1} \text { on } \Lambda_{1}, \quad \text { where } \Theta_{t}=\left(\vartheta_{j}^{(k)}(t, \cdot)\right)_{k, j=0}^{3}, \\
\Phi & =\left(\phi_{k j}\right)_{k, j=0}^{3}=\Theta_{0}^{-1} \Theta_{1} e^{-z \Omega} .
\end{aligned}
$$

The functions $\phi_{k j}, k, j \in \mathbb{N}_{3}$, are analytic in $\Lambda_{1}^{ \pm}$and satisfy the relations

$$
\begin{aligned}
\left|\phi_{j j}(\lambda)\right| & \leq \frac{4}{3}, \quad\left|\phi_{k j}(\lambda)-\delta_{k j}\right| \leq \frac{\varkappa}{|z|}, \quad \lambda \in \Lambda_{3}, \\
\phi_{k j}(\lambda) & =O\left(z^{-2}\right), \quad k \neq j, \quad \phi_{j j}(\lambda)=e^{-\frac{\omega_{j}^{3} \hat{p}_{0}}{4 z}}+O\left(z^{-3}\right) \quad \text { as }|\lambda| \rightarrow \infty .
\end{aligned}
$$

Moreover, we have the asymptotic formula

$$
\Theta_{0}(\lambda)=U(\lambda)\left(I_{4}+O\left(\frac{1}{z}\right)\right) \text { as }|\lambda| \rightarrow \infty, \quad \text { where } U=\left(z^{k} \omega_{j}^{k}\right)_{k, j=0}^{3} .
$$


ii) The following asymptotic relations hold:

$$
\begin{aligned}
& \phi_{12}(\lambda)=-2 \xi^{2} \widehat{\widehat{p_{n}^{\prime}}}+O\left(\xi^{3}\right), \\
& \phi_{21}(\lambda)=-2 \xi^{2} \widehat{p_{n}^{\prime}}+O\left(\xi^{3}\right) \text { for } z=\pi n+O\left(n^{-1}\right), \\
& \phi_{01}(\lambda) \phi_{10}(\lambda)=2\left|\widehat{p_{n}^{\prime}}\right|^{2} \xi^{4}+O\left(\xi^{5}\right) \text { for } z=(1+i) \pi n+O\left(n^{-1}\right), \\
& \xi=\frac{1}{4 \pi n}, \quad n \rightarrow \infty .
\end{aligned}
$$

Remarks. 1) Relation (3.5) shows that the matrices $M$ and $\Phi e^{z \Omega}$ have the same eigenvalues.

2) If $p=q=0$, then $\Phi=I_{4}$. The function $T_{1}^{0}$ defined in (1.8) is expressed in terms of the solutions $\vartheta_{j}^{0}=e^{z t \omega_{j}}$ of the nonperturbed equation by the formula $T_{1}^{0}=\frac{1}{4} \sum_{0}^{3} \vartheta_{j}^{0}(1)=$ $\frac{1}{4} \sum_{0}^{3} e^{z \omega_{j}}$.

We introduce the functions

$$
T=4 T_{1}^{2}-T_{2}, \quad T^{0}=4\left(T_{1}^{0}\right)^{2}-T_{2}^{0}=1+2 \cosh z \cos z=\frac{1}{2} \sum_{0 \leq j<k \leq 3} e^{z\left(\omega_{j}+\omega_{k}\right)} .
$$

Lemma 3.2. The functions $T_{1}$ and $T$ satisfy the relations

$$
T_{1}=\frac{1}{4} \sum_{0}^{3} \phi_{j j} e^{z \omega_{j}}, \quad T=\frac{1}{2} \sum_{0 \leq j<k \leq 3} v_{j k} e^{z\left(\omega_{j}+\omega_{k}\right)},
$$

where $v_{j k}=\phi_{j j} \phi_{k k}-\phi_{j k} \phi_{k j}$,

$$
\left|T_{1}(\lambda)-T_{1}^{0}(\lambda)\right| \leq \frac{\varkappa}{|z|} e^{x}, \quad\left|T(\lambda)-T^{0}(\lambda)\right| \leq \frac{9 \varkappa}{|z|} e^{x+|y|} \text { for } \lambda \in \Lambda_{3} .
$$

Proof. Relation (3.5) implies that $T_{1}=\frac{1}{4} \operatorname{Tr} M=\frac{1}{4} \operatorname{Tr} \Phi e^{z \Omega}$, whence the first identity in (3.12) follows. Moreover,

$$
T_{2}=\frac{1}{4} \operatorname{Tr} M^{2}=\frac{1}{4} \operatorname{Tr}\left(\Phi e^{z \Omega}\right)^{2}=\frac{1}{4}\left(\sum_{j=0}^{3} \phi_{j j}^{2} e^{2 z \omega_{j}}+2 \sum_{0 \leq j<k \leq 3} \phi_{j k} \phi_{k j} e^{z\left(\omega_{j}+\omega_{k}\right)}\right) .
$$

Substituting this and the first relation from (3.12) in (3.11), we obtain the second relation in (3.12). Relations (3.12) imply that

$$
T_{1}-T_{1}^{0}=\frac{1}{4} \sum_{k=0}^{3} e^{z \omega_{k}}\left(\phi_{k k}-1\right), \quad T-T^{0}=\frac{1}{2} \sum_{0 \leq j<k \leq 3} e^{z\left(\omega_{j}+\omega_{k}\right)}\left(v_{j k}-1\right) .
$$

Inequalities (3.6) and $\left|e^{z \omega_{k}}\right| \leq e^{x}$ provide the first estimate in (3.13). Inequalities (3.6) yield

$$
\left|v_{j k}-1\right| \leq\left|\phi_{j j}-1\right|+\left|\phi_{k k}-1\right|+\left|\phi_{j j}-1\right|\left|\phi_{k k}-1\right|+\left|\phi_{j k}\right|\left|\phi_{k j}\right| \leq 2 \frac{\varkappa}{|z|}\left(1+\frac{\varkappa}{|z|}\right) \leq \frac{3 \varkappa}{|z|}
$$

on $\Lambda_{3}$. Using the estimates $\left|e^{z\left(\omega_{j}+\omega_{k}\right)}\right| \leq e^{x+|y|}$, we obtain the second estimate in (3.13).

We need simple identities, which follow from (1.3), (1.4), and (1.5):

$$
\begin{aligned}
& \Delta_{1}^{2}+\Delta_{2}^{2}=1+T_{2}, \quad \Delta_{1} \Delta_{2}=2 T_{1}^{2}-\frac{T_{2}+1}{2}=\frac{T-1}{2} \\
& D_{ \pm}=\left(T_{1} \mp 1\right)^{2}-\rho=\frac{\left(2 T_{1} \mp 1\right)^{2}-T_{2}}{2}=\frac{T \mp 4 T_{1}+1}{2}, \quad D_{+}-D_{-}=-4 T_{1} .
\end{aligned}
$$


We introduce the domains

$$
\mathcal{D}_{n}=\left\{\lambda \in \mathbb{C}:\left|\lambda^{1 / 4}-(1 \pm i) \pi n\right|<\frac{\pi}{2 \sqrt{2}}\right\}, \quad n \geq 0, \quad \mathcal{D}=\mathbb{C} \backslash \bigcup_{n \geq 0} \overline{\mathcal{D}_{n}} .
$$

Lemma 3.3. i) The function $\rho=\frac{1-T}{2}+T_{1}^{2}$ (see (1.3)) is entire, real on $\mathbb{R}$, and the following relations are valid:

$$
\begin{aligned}
& \left|\rho(\lambda)-\rho^{0}(\lambda)\right| \leq \frac{3 \varkappa}{|z|_{1}} e^{2(x+\varkappa)}, \quad \lambda \in \mathbb{C}, \\
& \rho(\lambda)=\rho^{0}(\lambda)\left(1+O\left(z^{-1}\right)\right) \text { as }|\lambda| \rightarrow \infty, \quad \lambda \in \mathcal{D}, \\
& \rho(\lambda)=\frac{1}{16}\left(4 e^{(1-i) z} \phi_{01}(\lambda) \phi_{10}(\lambda)+\left(\phi_{00}(\lambda) e^{z}-\phi_{11}(\lambda) e^{-i z}\right)^{2}+O(1)\right), \\
& \quad|\lambda| \rightarrow \infty, \quad x-y \leq \pi .
\end{aligned}
$$

ii) For every integer $N>n_{0}$ with a certain $n_{0} \geq 1$, the function $\rho$ has precisely $2 N+1$ zeros (counted with multiplicity) in the disk $\left\{\lambda:|\lambda|<4\left(\pi\left(N+\frac{1}{2}\right)\right)^{4}\right\}$ and for every $n>N$ precisely two zeros (counted with multiplicity) in the domain $\mathcal{D}_{n}$. The function $\rho$ has no other zeros.

Proof. i) By Lemma 2.1, the functions $T_{\nu}, \nu=1,2$, are entire and real on $\mathbb{R}$. Therefore, $\rho$ is entire and real on $\mathbb{R}$. Next, we have

$$
\rho^{0}=\frac{T_{2}^{0}+1}{2}-\left(T_{1}^{0}\right)^{2}=-\sinh ^{2} \frac{(1-i) z}{2} \sin ^{2} \frac{(1-i) z}{2} .
$$

The first relation in (3.19) yields

$$
\left|\rho(\lambda)-\rho^{0}(\lambda)\right| \leq \frac{\left|T_{2}(\lambda)-T_{2}^{0}(\lambda)\right|}{2}+\left|T_{1}(\lambda)-T_{1}^{0}(\lambda)\right|\left|T_{1}(\lambda)+T_{1}^{0}(\lambda)\right|, \quad \lambda \in \mathbb{C} .
$$

Inequalities (2.3) imply (3.16). Using (3.19) and the inequality $e^{|y|}<4|\sin z|$ for $|z-\pi n| \geq \frac{\pi}{4}, n \in \mathbb{Z}$ (see 29$]$ ), we obtain

$$
\left|\rho^{0}(\lambda)\right|>\frac{1}{16} e^{2\left|\operatorname{Im} \frac{(1-i) z}{2}\right|+2\left|\operatorname{Im} \frac{i(1-i) z}{2}\right|}=\frac{1}{16} e^{|y+x|+|y-x|}=\frac{e^{2 x}}{16}, \quad \lambda \in \mathcal{D} .
$$

Then (3.16) follows from (3.17). Relation (3.12) provides

$$
T_{1}(\lambda)=\frac{1}{4}\left(\phi_{00}(\lambda) e^{z}+\phi_{11}(\lambda) e^{-i z}+O\left(e^{-x}\right)\right), \quad T(\lambda)=\frac{1}{2}\left(e^{(1-i) z} v_{01}(\lambda)+O(1)\right)
$$

as $|\lambda| \rightarrow \infty, x-y \leq \pi$. The last relation in (3.14) implies that

$$
\begin{aligned}
\rho & =\frac{1}{2}\left(1-\frac{1}{2}\left[e^{(1-i) z} v_{01}+O(1)\right]\right)+\frac{1}{4^{2}}\left(\phi_{00} e^{z}+\phi_{11} e^{-i z}+O\left(e^{-x}\right)\right)^{2} \\
& =\frac{1}{16}\left(-4 e^{(1-i) z} v_{01}+\phi_{00}^{2} e^{2 z}+\phi_{11}^{2} e^{-2 i z}+2 \phi_{00} \phi_{11} e^{(1-i) z}+O(1)\right) \\
& =\frac{1}{16}\left(4 e^{(1-i) z} \phi_{01} \phi_{10}+\phi_{00}^{2} e^{2 z}+\phi_{11}^{2} e^{-2 i z}-2 \phi_{00} \phi_{11} e^{(1-i) z}+O(1)\right),
\end{aligned}
$$

as $|\lambda| \rightarrow \infty, x-y \leq \pi$, which yields (3.18).

ii) Consider the contour $C_{0}(r)=\left\{\lambda:\left|\lambda^{1 / 4}\right|=\pi r\right\}$. Let $N_{1}>N$ be any integer. Consider the contours $C_{0}\left(N+\frac{1}{2}\right), C_{0}\left(N_{1}+\frac{1}{2}\right), \partial \mathcal{D}_{n}, n>N$. Then (3.16) and (3.20) provide

$$
\left|\rho(\lambda)-\rho^{0}(\lambda)\right| \leq o(1) e^{2 x}<\left|\rho^{0}(\lambda)\right|
$$

on all contours. By the Rouché theorem, $\rho$ has as many zeros counted with multiplicity as $\rho^{0}$ has in each of the bounded domains and in the remaining unbounded domain. Since $\rho^{0}$ has precisely one simple zero for $\lambda=0$ and precisely one zero of multiplicity 2 
at $-4(\pi n)^{4}, n \geq 1$, and since $N_{1}>N$ can be chosen arbitrarily large, we obtain the required statement.

Remark. Since the nonreal resonances counted with multiplicity form pairs of complex conjugate points, we can enumerate them in such a way that $\operatorname{Im} r_{n}^{+} \geq 0, \operatorname{Im} r_{n}^{-} \leq 0$ for all $n \in \mathbb{N}$.

Lemma 3.4. The functions $D_{ \pm}$are entire, real on $\mathbb{R}$, and the following estimate is valid:

$$
\left|D_{ \pm}(\lambda)-D_{ \pm}^{0}(\lambda)\right| \leq \frac{7 \varkappa}{|z|} e^{x+|y|}, \quad \lambda \in \Lambda_{4} .
$$

Moreover, for every integer $N>n_{0}$ with a certain $n_{0} \geq 1$, the function $D_{+}$has precisely $2 N+1$ zeros counted with multiplicity in the domain $\left\{|\lambda|^{1 / 4}<2 \pi\left(N+\frac{1}{2}\right)\right\}$, and for each $n>N$, the function $D_{+}$has precisely two zeros in the domain $\left\{\left|\lambda^{1 / 4}-2 \pi n\right|<\frac{\pi}{2}\right\}$, whereas the function $D_{-}$has precisely two zeros in the domain $\left\{\left|\lambda^{1 / 4}-\pi(2 n+1)\right|<\frac{\pi}{2}\right\}$. These functions have no other zeros.

Proof. Relations 3.15 imply that $D_{ \pm}$are entire, real on $\mathbb{R}$, and

$$
\left|D_{ \pm}-D_{ \pm}^{0}\right| \leq \frac{\left|T-T^{0}\right|+4\left|T_{1}-T_{1}^{0}\right|}{2} \leq\left(9 e^{x+|y|}+4 e^{x}\right) \frac{\varkappa}{2|z|},
$$

whence (3.21) follows. Let $N^{\prime}>N$ be any integer. Let $\lambda$ belong to the contours $C_{0}(2 N+1), C_{0}\left(2 N^{\prime}+1\right), C_{2 n}\left(\frac{1}{2}\right),|n|>N$, where $C_{n}(r)=\left\{\lambda:\left|\lambda^{1 / 4}-\pi n\right|=\pi r\right\}$, $r>0$. Note that $e^{\frac{1}{2}|y|}<4\left|\sin \frac{z}{2}\right|, e^{\frac{1}{2} x}<4\left|\sinh \frac{z}{2}\right|, z=\lambda^{1 / 4}$, on all contours. Then $e^{\frac{1}{2}(x+|y|)}<16\left|\sin \frac{z}{2} \sinh \frac{z}{2}\right|$ and (3.21) on all contours yield

$$
\left|D_{+}(\lambda)-4 \sin ^{2} \frac{z}{2} \sinh ^{2} \frac{z}{2}\right| \leq o(1) e^{x+|y|}<\left|4 \sin ^{2} \frac{z}{2} \sinh ^{2} \frac{z}{2}\right| .
$$

By the Rouché theorem, $D_{+}$has as many zeros as $\sin ^{2} \frac{z}{2} \sinh ^{2} \frac{z}{2}$ has in each bounded domain and in the remaining unbounded domain. Since $\sin ^{2} \frac{z}{2} \sinh ^{2} \frac{z}{2}$ has precisely one simple zero at $\lambda=0$ and precisely one zero of multiplicity 2 at $(2 \pi n)^{4}, n \geq 1$, and since $N^{\prime}>N$ can be chosen arbitrarily large, we obtain the statement for $D_{+}$. For $D_{-}$, it is proved similarly.

Proof of Theorem 1.1 i), ii). Following the line of argument in [2, 5, we prove the analyticity of $\Delta$ on the surface $\mathscr{R}$, relations (1.5), and statement ii). Inequalities (3.13) provide the relation $T_{1}(\lambda)=T_{1}^{0}(\lambda)+e^{x} O\left(z^{-1}\right),|\lambda| \rightarrow \infty$. Substituting these relations and 3.17) in (1.4), we get

$$
\Delta_{1}(\lambda)=T_{1}^{0}(\lambda)+\sqrt{\rho^{0}(\lambda)}+e^{x} O\left(z^{-1}\right) \text { as }|\lambda| \rightarrow \infty, \quad \lambda \in \mathcal{D} .
$$

Using the relation $\Delta_{1}^{0}=T_{1}^{0}+\sqrt{\rho^{0}}=\cosh z$ (see (1.8)), we arrive at the first relations in (1.6).

Inequalities (3.13) yield the relation $T(\lambda)=T^{0}(\lambda)+e^{x+|y|} O\left(z^{-1}\right)$ as $|\lambda| \rightarrow \infty$. Substituting these relations and (1.6) in the relation $\Delta_{2}=\frac{T-1}{2 \Delta_{1}}$ (see (3.14)), we obtain

$$
\Delta_{2}(\lambda)=\frac{T^{0}(\lambda)-1}{2 \cosh z}+e^{|y|} O\left(z^{-1}\right) \text { as }|\lambda| \rightarrow \infty, \quad \lambda \in \mathcal{D} .
$$

Next, the identity $\Delta_{2}^{0}=\frac{T^{0}-1}{2 \cosh z}=\cos z$ implies the second relations in (1.6). 


\section{$\S 4$. Floquet solutions}

In this section, we study some properties of the Floquet solutions of equation (1.1). Here it is convenient to assume that $\arg \lambda \in[0,2 \pi), \arg z \in\left[0, \frac{\pi}{2}\right)$. We introduce the domains

$$
\begin{array}{r}
\mathscr{U}_{R}=\left\{\lambda:|\lambda|>R,|z-(1+i) \pi n|>\frac{\pi}{2 \sqrt{2}},|z-\pi n|>\frac{\pi}{2} \text { for all } n \in \mathbb{N}\right\}, \\
R>0 .
\end{array}
$$

The relations (1.6) show that

$$
\tau_{1}(\lambda)=e^{z}\left(1+O\left(\frac{1}{z}\right)\right), \quad \tau_{2}(\lambda)=e^{-i z}\left(1+O\left(\frac{1}{z}\right)\right), \quad|\lambda| \rightarrow \infty, \quad \lambda \in \mathscr{U}_{1} .
$$

Recall that two (or more) multipliers coincide for some $\lambda \in \mathbb{C}$ if and only if $\lambda$ is a 2-periodic eigenvalue or a resonance. Lemmas 3.3, ii) and 3.4 prove that for $R>0$ sufficiently large, the domain $\mathscr{U}_{R}$ contains neither 2-periodic eigenvalues nor resonances. Therefore, if $\lambda \in \mathscr{U}_{R}$ for such an $R$, then the four multipliers $\tau_{\nu}^{ \pm 1}(\lambda), \nu=1,2$, are distinct.

In the sequel we shall need a more accurate description of the multipliers in the domain

$$
\mathscr{L}_{R}=\{\lambda \in \mathbb{C}:|\lambda|>R, \operatorname{Re} \lambda<0\}, \quad R>0 .
$$

Equation (1.2) determines a function $\tau(\lambda)$ analytic on a 4-sheeted Riemann surface (some properties of the corresponding surface for an operator of any even order were described by Tkachenko in [33]). The asymptotic formula (4.1) determines single-valued functions $\tau_{\nu}$ for $\nu=1,2$ in the domain $\mathscr{U}_{R}$ with $R>0$ sufficiently large. The branch points of the multipliers in the domain $\mathscr{L}_{R}$ may coincide only with the resonances $r_{n}^{ \pm}$. The functions $\tau_{\nu}$ are continued as single-valued functions from the domain $\mathscr{U}_{R} \cup \mathscr{L}_{R}$ to the domain $\widetilde{\mathscr{L}}_{R}=\mathscr{L}_{R} \backslash \bigcup_{n \geq 1}\left[r_{n}^{-}, r_{n}^{+}\right]$.

Remark. Such a drawing of the cuts for separating single-valued branches of the function $\tau$ differs from that used in other parts of the present paper. It is convenient for the analysis of solutions of equation (1.1) in a neighborhood of resonances.

The asymptotic formula (4.1) shows that $\left|\tau_{1}(\lambda)\right|>1$ and $\left|\tau_{2}(\lambda)\right|>1$ for all $\lambda \in$ $\mathscr{U}_{R} \cap \mathscr{L}_{R}$ with $R>0$ sufficiently large. In the next lemma we establish that these inequalities are valid for all $\lambda \in \widetilde{\mathscr{L}}_{R}$.

Lemma 4.1. The following inequalities hold true:

$$
\left|\tau_{\nu}(\lambda)\right|>1 \text { for all } \nu=1,2, \quad \lambda \in \widetilde{\mathscr{L}}_{R}=\mathscr{L}_{R} \backslash \bigcup_{n \geq 1}\left[r_{n}^{-}, r_{n}^{+}\right] .
$$

The functions $\tau_{1}+\tau_{2}$ and $\tau_{1} \tau_{2}$ have analytic continuations from the domain $\widetilde{\mathscr{L}}_{R}$ to the domain $\mathscr{L}_{R}$. Moreover, these functions are real on the interval $(-\infty,-R)$.

Proof. We prove (4.2) for $\tau_{1}$. The proof for $\tau_{2}$ is similar. Suppose the contrary. Let $\tau_{1}$ satisfy the condition $\left|\tau_{1}\left(\lambda_{0}\right)\right|<1$ for some $\lambda_{0} \in \mathscr{L}_{R}$. Then $\lambda_{0} \in \mathcal{D}_{n}=\{\lambda \in \mathbb{C}$ : $\left.|z-(1+i) \pi n|<\frac{\pi}{2 \sqrt{2}}\right\}$ with some (large) $n \geq 1$. Assume for definiteness that $\operatorname{Im} \lambda_{0} \geq 0$; the case where $\operatorname{Im} \lambda_{0}<0$ is considered similarly. Let $\lambda_{1}$ lie outside the domain $\mathcal{D}_{n}$ but sufficiently close to it, and let $\operatorname{Im} \lambda_{1}>0$. Then $\lambda_{1} \in \mathscr{U}_{R} \cap \mathscr{L}_{R}$ and, as the asymptotic formula (4.1) shows, $\left|\tau_{1}\left(\lambda_{1}\right)\right|>1$. By Lemma 3.3, ii), inside $\mathcal{D}_{n}$ there are precisely two resonances $r_{n}^{-}$and $r_{n}^{+}$and there are no other resonances in some neighborhood of $\mathcal{D}_{n}$. Therefore we may draw a curve $\ell \subset \mathbb{C}_{+} \cap \widetilde{\mathscr{L}}_{R}$ that joins the points $\lambda_{0}$ and $\lambda_{1}$. The multiplier $\tau_{1}$ depends on $\lambda$ continuously on the curve $\ell$. Therefore, there is a point $\lambda_{2}$ on this curve such that $\left|\tau_{1}\left(\lambda_{2}\right)\right|=1$ and $\operatorname{Im} \lambda_{2}>0$. Then $\lambda_{2}$ is a point of the spectrum 
of the operator $H$. But this contradicts the fact that the spectrum is real, which proves (4.2).

Estimate (4.2) shows that $\tau_{1} \neq \tau_{1}^{-1}$ and $\tau_{1} \neq \tau_{2}^{-1}$ in $\mathscr{L}_{R}$. Therefore, all resonances lying in $\mathscr{L}_{R}$ are zeros of the function $\tau_{1}-\tau_{2}$. The multiplicity of these resonances is equal to 1 or 2 . If a resonance has multiplicity 2 , then the functions $\tau_{1}$ and $\tau_{2}$ are analytic in its neighborhood. If a resonance has multiplicity 1 , then it is a simple (of the square root type) branch point of the four-sheeted Riemann surface of multipliers in which the sheets that correspond to the multipliers $\tau_{1}$ and $\tau_{2}$, as well as to $\tau_{1}^{-1}$ and $\tau_{2}^{-1}$, merge pairwise. The functions $\tau_{1}+\tau_{2}$ and $\tau_{1} \tau_{2}$ can be continued analytically along any curve in $\mathscr{L}_{R}$ not passing through resonances. Since the values of $\tau_{1}(\lambda)$ and $\tau_{2}(\lambda)$ may only be permuted under analytic continuation along any closed curve in $\mathscr{L}_{R}$, the functions $\tau_{1}+\tau_{2}$ and $\tau_{1} \tau_{2}$ are single-valued in $\mathscr{L}_{R}$. This implies that the functions $\tau_{1}+\tau_{2}$ and $\tau_{1} \tau_{2}$ admit analytic continuation to the entire domain $\mathscr{L}_{R}$.

Let $\lambda<-R$. Estimate (4.2) shows that $\left|\tau_{\nu}(\lambda)\right|>1$ and $\left|\tau_{\nu}^{-1}(\lambda)\right|<1$. Then either the two multipliers $\tau_{1}(\lambda)$ and $\tau_{2}(\lambda)$ are real, or $\tau_{1}(\lambda)=\bar{\tau}_{2}(\lambda)$. This implies that $\tau_{1}(\lambda)+\tau_{2}(\lambda)$ and $\tau_{1}(\lambda) \tau_{2}(\lambda)$ are real.

We define functions $m_{j}(\lambda, \tau),(j, \lambda, \tau) \in \mathbb{N}_{3} \times \mathbb{C}^{2}$, by the relations

$$
\begin{aligned}
& m_{0}=1, \quad m_{j}=-\frac{c_{j}}{c}, \quad j=1,2,3, \\
& \text { where } c(\lambda, \tau)=\operatorname{det}\left(\begin{array}{ccc}
\varphi_{1} & \varphi_{2} & \varphi_{3} \\
\varphi_{1}^{\prime}-\tau & \varphi_{2}^{\prime} & \varphi_{3}^{\prime} \\
\varphi_{1}^{\prime \prime} & \varphi_{2}^{\prime \prime}-\tau & \varphi_{3}^{\prime \prime}
\end{array}\right)(1, \lambda),
\end{aligned}
$$

and where each of the functions $c_{j}, j=1,2,3$, is obtained by replacement of the $j$ th column in the determinant for $c$ in (4.3) by the column $\left(\varphi_{0}-\tau, \varphi_{0}^{\prime}, \varphi_{0}^{\prime \prime}\right)^{\top}$.

Lemma 4.2. i) Let $\lambda \in \mathscr{U}_{R}$ with some large $R>0$. Then there exist Floquet solutions $\psi_{\nu}^{ \pm}(t, \lambda), \nu=1,2, t \in \mathbb{R}$, of equation (1.1) given by the relations

$$
\psi_{\nu}^{ \pm}(t, \lambda)=\psi\left(t, \lambda, \tau_{\nu}^{ \pm 1}(\lambda)\right), \quad \text { where } \quad \psi(t, \lambda, \tau)=\sum_{k=0}^{3} m_{k}(\lambda, \tau) \varphi_{k}(t, \lambda) .
$$

These solutions satisfy the conditions

$$
\psi_{\nu}^{ \pm}(t+1, \lambda)=\tau_{\nu}^{ \pm 1}(\lambda) \psi_{\nu}^{ \pm}(t, \lambda) \quad \text { for all } \quad t \in \mathbb{R}, \quad \psi_{\nu}^{ \pm}(0, \lambda)=1 .
$$

ii) The functions $c_{\nu}^{ \pm}=c\left(\cdot, \tau_{\nu}^{ \pm 1}(\cdot)\right)$ do not vanish in the domain $\mathscr{U}_{R}$. We have the following asymptotic formulas:

$$
\begin{aligned}
& \psi_{1}^{ \pm}(t, \lambda)=e^{ \pm z t}\left(1+O\left(\frac{1}{z}\right)\right), \\
& \psi_{2}^{ \pm}(t, \lambda)=e^{\mp i z t}\left(1+O\left(\frac{1}{z}\right)\right) \quad \text { as } \quad|\lambda| \rightarrow \infty, \quad \lambda \in \mathscr{U}_{R},
\end{aligned}
$$

uniformly with respect to $t \in[0,1]$. These formulas can be differentiated three times with respect to $t$, retaining an estimate uniform with respect to $t$ of the remainder term.

iii) Let the function $f$ satisfy equation (1.1) for some $\lambda \in \mathscr{U}_{R}$ and conditions (4.5). Then $f=\psi_{\nu}^{ \pm}$for some $\nu=1,2$.

iv) The functions $c_{\nu}^{ \pm}, \nu=1,2$, admit analytic continuation to the domain $\widetilde{\mathscr{L}}_{R}$. If $\lambda \in \widetilde{\mathscr{L}}_{R}$ is not a zero of the function $c_{\nu}^{ \pm}$for $\nu=1$ or 2 , then $\psi_{\nu}^{ \pm}(t, \cdot)$ is analytic at the point $\lambda$.

v) If $\lambda \in \widetilde{\mathscr{L}_{R}}$ is a zero of the function $c_{\nu}^{ \pm}$for $\nu=1$ or 2 , then $\lambda$ is an eigenvalue of the multipoint Dirichlet problem $y(0)=y(1)=y(2)=y(3)=0$ for equation (1.1). 
Proof. i) Since $\lambda$ is not a branch point of the function $\tau$, the monodromy matrix has precisely four simple eigenvalues $\tau_{1}^{ \pm 1}(\lambda), \tau_{2}^{ \pm 1}(\lambda)$. Note that the vector $m_{\nu}^{ \pm}=\left(m_{i, \nu}^{ \pm}\right)_{i=0}^{3}$, where

$$
m_{i, \nu}^{ \pm}(\lambda)=m_{i}\left(\lambda, \tau_{\nu}^{ \pm 1}(\lambda)\right), \quad \lambda \in \mathbb{C}, \quad i=1,2,3, \quad \nu=1,2,
$$

and where the $m_{i}$ are defined by relations (4.3), is an eigenvector of the matrix $M$ corresponding to the eigenvalue $\tau_{\nu}^{ \pm 1}$ :

$$
M m_{\nu}^{ \pm}=\tau_{\nu}^{ \pm 1} m_{\nu}^{ \pm}, \quad m_{\nu}^{ \pm}=\left(m_{i, \nu}^{ \pm}\right)_{i=0}^{3}, \quad m_{0, \nu}^{ \pm}=1, \quad \nu=1,2 .
$$

The functions $\psi_{\nu}^{ \pm}$defined by (4.4) satisfy equation (1.1). We show that they satisfy conditions (4.5) as well. The initial conditions for the functions $\varphi_{j}$ yield $\psi_{\nu}^{ \pm}(0, \lambda)=1$. Using the standard properties of the matrix-valued function $\mathcal{M}(t, \lambda)=\left(\varphi_{j}^{(k)}(t, \lambda)\right)_{k, j=0}^{3}$, $(t, \lambda) \in \mathbb{R} \times \mathbb{C}$ (see, e.g., [34, Chapter II.1.2]), we have

$$
\Psi_{\nu}^{ \pm}(t+1, \cdot)=\mathcal{M}(t+1, \cdot) m_{\nu}^{ \pm}=\mathcal{M}(t, \cdot) M m_{\nu}^{ \pm}=\tau_{\nu}^{ \pm 1} \mathcal{M}(t, \cdot) m_{\nu}^{ \pm}=\tau_{\nu}^{ \pm 1} \Psi_{\nu}^{ \pm}(t, \cdot),
$$

where $\Psi_{\nu}^{ \pm}=\left(\left(\psi_{\nu}^{ \pm}\right)^{(k)}\right)_{k=0}^{3}$. This implies that $\psi_{\nu}^{ \pm}(t+1, \lambda)=\tau_{\nu}^{ \pm 1}(\lambda) \psi_{\nu}^{ \pm}(t, \lambda)$. Thus, the functions $\psi_{\nu}^{ \pm}$satisfy conditions (4.5).

ii) We show that the functions $c_{\nu}^{ \pm}=c\left(\cdot, \tau_{\nu}^{ \pm 1}(\cdot)\right)$ do not vanish in the domain $\mathscr{U}_{R}$. Note that

$$
\begin{aligned}
& c_{\nu}^{ \pm}(\lambda)=0 \text { if and only if the equation } M(\lambda) h=\tau_{\nu}^{ \pm 1}(\lambda) h \\
& \quad \text { has a solution of the form } h=\left(h_{i}\right)_{1}^{4} \neq 0 \text { with } h_{1}=0 .
\end{aligned}
$$

In what follows we consider $c_{1}^{+}$. The proof for the other functions $c_{\nu}^{ \pm}$is similar. Let $\lambda \in \mathscr{U}_{R}$, and let $h=\left(h_{i}\right)_{1}^{4}=m_{1}^{+}$be an eigenvector of the matrix $M(\lambda)$ that corresponds to the simple eigenvalue $\tau_{1}(\lambda)$. Substituting (3.5) in the relation $M(\lambda) h=\tau_{1}(\lambda) h$ and using the asymptotic formulas (3.7) and (4.1), we obtain $e^{z \Omega} \widetilde{h}=e^{z}\left(I_{4}+O\left(\frac{1}{z}\right)\right) \widetilde{h}$, where $\widetilde{h}=\left(\widetilde{h}_{i}\right)_{1}^{4}=\Theta_{0}^{-1} h$. Hence, we get

$$
\left(e^{-z} e^{z \Omega}-I_{4}+O\left(\frac{1}{z}\right)\right) \tilde{h}=0 \quad \text { as }|\lambda| \rightarrow \infty .
$$

We have $e^{-z} e^{z \Omega}-I_{4}=\operatorname{diag}\left(0, e_{1}, e_{2}, e_{3}\right)$, where $e_{j}=e^{-z\left(1-\omega_{j}\right)}-1, j=1,2,3$.

We show that

$$
\left|e_{j}(\lambda)\right| \geq \frac{1}{2} \quad \text { for all } j=1,2,3, \lambda \in \mathscr{U}_{R} .
$$

We prove (4.11) for $j=1, \operatorname{Im} \lambda \geq 0$. The proof for the other cases is similar. From (3.1) we have $e_{1}(\lambda)=e^{-(1+i) z}-1$. Using the relations

$$
\left|e^{-(1+i) \frac{z}{2}}\right|=e^{\frac{y-x}{2}}, \quad\left|\sinh \frac{(1+i) z}{2}\right| \geq \frac{1}{4} e^{\mid \operatorname{Re} \frac{(1+i) z}{2}} \mid \geq \frac{1}{4} e^{\frac{x-y}{2}},
$$

where $x=\operatorname{Re} z, y=\operatorname{Im} z$ (see [29]), we get

$$
\left|e_{j}(\lambda)\right|=\left|e^{-(1+i) z}-1\right|=2\left|e^{-(1+i) \frac{z}{2}} \sinh \frac{(1+i) z}{2}\right| \geq \frac{1}{2},
$$

which proves (4.11) for $e_{1}(\lambda), \operatorname{Im} \lambda \geq 0$.

Multiplying the two parts of (4.10) by the matrix $\operatorname{diag}\left(1, e_{1}^{-1}, e_{2}^{-1}, e_{3}^{-1}\right)$, in view of (4.11) we obtain

$$
\left(J+O\left(\frac{1}{z}\right)\right) \widetilde{h}=0, \quad \text { where } J=\operatorname{diag}(0,1,1,1) .
$$

Now we prove that $\widetilde{h}_{1} \neq 0$. Suppose the contrary. Let $\widetilde{h}_{1}=0$. Then, multiplying the two parts of (4.12) by the vector $(0,1,1,1)^{\top}$, we arrive at $\left(I_{3}+O\left(\frac{1}{z}\right)\right)\left(\widetilde{h}_{i}\right)_{2}^{4}=0$, whence the relations $\widetilde{h}_{2}=\widetilde{h}_{3}=\widetilde{h}_{4}=0$ follow. Then $h=\Theta_{0} \widetilde{h}=0$. Since $h$ is an eigenvector, 
we obtain a contradiction. Consequently, $\widetilde{h}_{1} \neq 0$, and we can normalize the vector $\widetilde{h}$ in such a way that $\widetilde{h}_{1}=1$. Again, multiplying the two parts of (4.12) by the vector $(0,1,1,1)^{\top}$, we obtain $\left(I_{3}+O\left(\frac{1}{z}\right)\right)\left(\widetilde{h}_{i}\right)_{2}^{4}=O\left(\frac{1}{z}\right)$, which implies that all the quantities $\widetilde{h}_{2}, \widetilde{h}_{3}, \widetilde{h}_{4}$ have asymptotic behavior $O\left(\frac{1}{z}\right)$ and then $\widetilde{h}=\left(1, O\left(\frac{1}{z}\right), O\left(\frac{1}{z}\right), O\left(\frac{1}{z}\right)\right)^{\top}$. The asymptotic formula (3.8) yields

$$
h=\Theta_{0}(\lambda) \tilde{h}=U(\lambda)\left(I_{4}+O\left(\frac{1}{z}\right)\right) \widetilde{h}=\left(\begin{array}{c}
1 \\
z\left(1+O\left(\frac{1}{z}\right)\right) \\
z^{2}\left(1+O\left(\frac{1}{)}\right)\right) \\
z^{3}\left(1+O\left(\frac{1}{z}\right)\right)
\end{array}\right) \text { as }|\lambda| \rightarrow \infty, \lambda \in \mathscr{U}_{R} .
$$

Thus, $h_{1} \neq 0$. From (4.9) it follows that $c_{1}^{+}(\lambda) \neq 0$.

We prove the asymptotic formulas (4.6) for $\psi_{1}^{+}$. The proof for the other functions $\psi_{\nu}^{ \pm}$is similar. The asymptotic formula (4.13) yields $m_{1}^{+}=h=\left(1, z\left(1+O\left(\frac{1}{z}\right)\right)\right.$, $\left.z^{2}\left(1+O\left(\frac{1}{z}\right)\right), z^{3}\left(1+O\left(\frac{1}{z}\right)\right)\right)^{\top}$. Substituting this expression in (4.4), we see that

$$
\begin{aligned}
\psi_{1}^{+}(t, \lambda)=\varphi_{0}(t, \lambda) & +z\left(1+O\left(\frac{1}{z}\right)\right) \varphi_{1}(t, \lambda) \\
& +z^{2}\left(1+O\left(\frac{1}{z}\right)\right) \varphi_{2}(t, \lambda)+z^{3}\left(1+O\left(\frac{1}{z}\right)\right) \varphi_{3}(t, \lambda)
\end{aligned}
$$

as $|\lambda| \rightarrow \infty$, uniformly with respect to $t \in[0,1]$. Estimates (2.2) show that $\varphi_{k}(t, \lambda)=$ $\varphi_{k}^{0}(t, \lambda)\left(1+O\left(\frac{1}{z}\right)\right)$ as $|\lambda| \rightarrow \infty$, uniformly with respect to $t \in[0,1]$ for all $k \in \mathbb{N}_{3}$, and these asymptotic formulas can be differentiated three times with respect to $t$, retaining the estimate uniform with respect to $t$ of the remainder term. Substituting these asymptotic formulas in (4.14) and using (2.1), we get (4.6) for $\psi_{1}^{+}$.

iii) The function $\psi=f-\psi_{\nu}^{ \pm}$satisfies the conditions $\psi(t+1)=\tau \psi(t), \psi(0)=0$ for all $t \in \mathbb{R}$. This implies the relation $\psi=\sum_{i=0}^{3} \tilde{m}_{i} \varphi_{i}$, where the first component $\tilde{m}_{0}$ of the vector $\left(\widetilde{m}_{i}\right)_{0}^{3} \in \mathbb{C}^{4}$ is equal to zero. Then

$$
\mathcal{M}(t, \cdot) M \tilde{m}_{i}=\mathcal{M}(t+1, \cdot) \tilde{m}_{i}=\Psi(t+1, \cdot)=\tau \Psi(t, \cdot)=\tau \mathcal{M}(t, \cdot) \tilde{m}_{i}, \quad \Psi=\left(\psi^{(k)}\right)_{k=0}^{3} .
$$

This means that the vector $\left(\tilde{m}_{i}\right)_{0}^{3}$ with $\tilde{m}_{0}=0$ is an eigenvector of the matrix $M$ that corresponds to the eigenvalue $\tau$. From (4.9), we obtain $c(\lambda, \tau(\lambda))=0$. Since the function $c(\cdot, \tau(\cdot))$ does not vanish in the domain $\mathscr{U}_{R}$, we see that $\left(\widetilde{m}_{i}\right)_{0}^{3}=0$ and $\psi=0$.

iv) Since the functions $\tau_{\nu}$ are analytic in the domain $\widetilde{\mathscr{L}}_{R}$, the functions $c_{\nu}^{ \pm}$admit analytic continuation to $\widetilde{\mathscr{L}}_{R}$. If $\lambda \in \widetilde{\mathscr{L}}_{R}$ is not a zero of the function $c_{\nu}^{ \pm}$, then, as relations (4.3) show, the functions $m_{k}\left(\cdot, \tau_{\nu}^{ \pm 1}(\cdot)\right), k=1,2,3$ are analytic at $\lambda$. Then relations (4.4) imply that $\psi_{\nu}^{ \pm}(t, \cdot)$ is analytic at the point $\lambda$.

v) Let $\lambda \in \widetilde{\mathscr{L}_{R}}$ be a zero of the function $c_{1}^{+}$. We show that $\lambda$ is an eigenvalue of the multipoint Dirichlet problem. The proof for other cases is similar. Since $\tau_{1}$ is an eigenvalue of the matrix $M$, it follows that at least one of the functions $c_{j}\left(\cdot, \tau_{1}(\cdot)\right), j=$ $1,2,3$, does not vanish at $\lambda$. Then the function $c_{1}^{+} \psi_{1}^{+}$is not equal to zero identically and vanishes at $t=0$. Moreover, this function satisfies the Floquet condition (4.5). This implies that it vanishes at all points $t=n, n \in \mathbb{Z}$. Consequently, $\lambda$ is an eigenvalue of the multipoint Dirichlet problem.

Consider the functions

$$
\psi_{\nu}(t, \lambda)=c_{\nu}^{+}(\lambda) \psi_{\nu}^{+}(t, \lambda), \quad \nu=1,2, \quad(t, \lambda) \in \mathbb{R} \times \mathbb{C},
$$

and the Wronskians

$$
w_{\ell n}=\psi_{1}^{(\ell)} \psi_{2}^{(n)}-\psi_{1}^{(n)} \psi_{2}^{(\ell)}, \quad 0 \leq \ell<n \leq 3 .
$$


Lemma 4.3. i) Every function $\psi_{\nu}(t, \cdot), \nu=1,2, t \in \mathbb{R}$, is analytic in the domain $\widetilde{\mathscr{L}}_{R}$ with $R>0$ sufficiently large. The following relations are valid:

$$
\psi_{\nu}(t, \lambda)=\sum_{j=0}^{3} \tau_{\nu}^{j}(\lambda) y_{j}(t, \lambda), \quad(t, \lambda) \in \mathbb{R} \times \widetilde{\mathscr{L}_{R}},
$$

where every function $y_{j}(\cdot, \lambda),(j, \lambda) \in \mathbb{N}_{3} \times \widetilde{\mathscr{L}}_{R}$, is a solution of equation (1.1). Moreover, every function $y_{j}(t, \cdot),(j, t) \in \mathbb{N}_{3} \times \mathbb{R}$, admits analytic continuation from $\widetilde{\mathscr{L}_{R}}$ to the entire complex plane, up to a function real on $\mathbb{R}$.

ii) Every function $w_{\ell n}^{2}(t, \cdot)$ admits analytic continuation from $\widetilde{\mathscr{L}}_{R}$ to $\mathscr{L}_{R}$, up to a function real on the interval $(-\infty,-R)$. The following relations are valid:

$$
w_{\ell n}(t, \lambda)=\left(\tau_{1}(\lambda)-\tau_{2}(\lambda)\right) A_{\ell n}(t, \lambda), \quad 0 \leq \ell<n \leq 3, \quad(t, \lambda) \in \mathbb{R} \times \widetilde{\mathscr{L}}_{R},
$$

where

$$
\begin{aligned}
A_{\ell n}=\varkappa_{01}^{(\ell n)} & +\left(\tau_{1}+\tau_{2}\right) \varkappa_{02}^{(\ell n)}+\left(\left(\tau_{1}+\tau_{2}\right)^{2}-\tau_{1} \tau_{2}\right) \varkappa_{03}^{(\ell n)} \\
& +\tau_{1} \tau_{2} \varkappa_{12}^{(\ell n)}+\tau_{1} \tau_{2}\left(\tau_{1}+\tau_{2}\right) \varkappa_{13}^{(\ell n)}+\left(\tau_{1} \tau_{2}\right)^{2} \varkappa_{23}^{(\ell n)},
\end{aligned}
$$

$0 \leq \ell<n \leq 3, \varkappa_{i j}^{(\ell n)}=y_{i}^{(\ell)} y_{j}^{(n)}-y_{j}^{(\ell)} y_{i}^{(n)}, 0 \leq i<j \leq 3$. Every function $\varkappa_{i j}^{(l n)}(t, \cdots)$, $0 \leq i<j \leq 3,0 \leq l<n \leq 3, t \in \mathbb{R}$, is an entire function real on $\mathbb{R}$. Every function $A_{\ell n}(t, \cdot), 0 \leq \ell<n \leq 3, t \in \mathbb{R}$, is analytic in the domain $\mathscr{L}_{R}$ and real on the interval $(-\infty,-R)$.

Proof. i) Substituting (4.3) and (4.4) in (4.15), we obtain

$$
\psi_{\nu}(t, \lambda)=-\operatorname{det}\left(\begin{array}{cccc}
\varphi_{0}(1, \lambda)-\tau_{\nu}(\lambda) & \varphi_{1}(1, \lambda) & \varphi_{2}(1, \lambda) & \varphi_{3}(1, \lambda) \\
\varphi_{0}^{\prime}(1, \lambda) & \varphi_{1}^{\prime}(1, \lambda)-\tau_{\nu}(\lambda) & \varphi_{2}^{\prime}(1, \lambda) & \varphi_{3}^{\prime}(1, \lambda) \\
\varphi_{0}^{\prime \prime}(1, \lambda) & \varphi_{1}^{\prime \prime}(1, \lambda) & \varphi_{2}^{\prime \prime}(1, \lambda)-\tau_{\nu}(\lambda) & \varphi_{3}^{\prime \prime}(1, \lambda) \\
\varphi_{0}(t, \lambda) & \varphi_{1}(t, \lambda) & \varphi_{2}(t, \lambda) & \varphi_{3}(t, \lambda)
\end{array}\right),
$$

$\nu=1,2,(t, \lambda) \in \mathbb{R} \times \widetilde{\mathscr{L}}_{R}$. This implies (4.17) and the fact that the $y_{j}$ are solutions of equation (1.1). Since the functions $\varphi_{j}(t, \cdot)$ are analytic and real on $\mathbb{R}$, we conclude that the $y_{j}(t, \cdot)$ have analytic extensions to $\mathbb{C}$ that are real on $\mathbb{R}$.

ii) Substituting (4.17) in (4.16), we get

$$
w_{\ell n}=\sum_{0 \leq i<j \leq 3}\left(\tau_{1}^{i} \tau_{2}^{j}-\tau_{1}^{j} \tau_{2}^{i}\right) \varkappa_{i j}^{(\ell n)}
$$

which implies (4.18). Since every function $y_{j}(t, \cdot)$ is entire and real on $\mathbb{R}$, it follows that every function $\varkappa_{i j}^{(\ell n)}(t, \cdot)$ is entire and real on $\mathbb{R}$. Then (4.19) and Lemma 4.1 imply that every function $A_{\ell n}, 0 \leq \ell<n \leq 3$, is analytic in $\lambda$ in the domain $\mathscr{L}_{R}$ and real on $(-\infty,-R)$. From (4.20) we get a similar assertion for $w_{\ell n}^{2}$.

Remark. An asymptotic investigation of the functions $A_{\ell n}(t, \cdot), t \in \mathbb{R}$, as $|\lambda| \rightarrow \infty, \lambda \in$ $\mathscr{L}_{R}$, shows that every such function has precisely one simple real zero in a neighborhood of every pair of resonances $r_{n}^{ \pm} \in \mathscr{L}_{R}$.

Lemma 4.4. Let $\lambda \in \mathscr{U}_{R}$ with $R>0$ sufficiently large, and let $\psi_{\nu}(\cdot, \lambda), \nu=1,2$, be two solutions of equation (1.1) determined by relation (4.15). Then (1.1) is equivalent to the equation

$$
f^{\prime \prime}+V(t, \lambda) f=0, \quad(t, \lambda) \in \mathbb{R} \times \mathscr{U}_{R},
$$


where the function $V$ is periodic in $t: V(t+1, \cdot)=V(t, \cdot)$ for all $t \in \mathbb{R}, V(\cdot, \lambda) \in L^{\infty}(0,1)$, and $V$ is given by the relation

$$
V=\frac{3}{2} \frac{w^{\prime \prime}}{w}-\frac{3}{4}\left(\frac{w^{\prime}}{w}\right)^{2}-\frac{w_{12}}{w}+p, \quad w=w_{01}=\psi_{1} \psi_{2}^{\prime}-\psi_{1}^{\prime} \psi_{2}, \quad w_{12}=\psi_{1}^{\prime} \psi_{2}^{\prime \prime}-\psi_{1}^{\prime \prime} \psi_{2}^{\prime} .
$$

Every function $V(t, \cdot), t \in \mathbb{R}$, is analytic in $\mathscr{U}_{R} \cap \mathscr{L}_{R}$ and has a meromorphic extension from this domain to $\mathscr{L}_{R}$. Every function $V(t, \lambda), t \in \mathbb{R}$, is real for $\lambda \in(-\infty,-R)$.

Proof. Let $\lambda \in \mathscr{U}_{R}$. Using the solutions $\psi_{1}$ and $\psi_{2}$ and standard substitutions, we reduce equation (1.1) to equation (4.21).

The asymptotic formulas (4.6) show that $\psi_{1}$ does not vanish for $\lambda \in \mathscr{U}_{R}$. Let $y$ be a solution of equation (1.1). We show that the function $u=\left(\frac{y}{\psi_{1}}\right)^{\prime}$ satisfies a linear equation of the third order. We have $y=\psi_{1} \widetilde{u}$, where $\widetilde{u}=\int u(t) d t$ and further $y^{\prime}=\psi_{1}^{\prime} \widetilde{u}+\psi_{1} u$, $y^{\prime \prime}=\psi_{1}^{\prime \prime} \widetilde{u}+2 \psi_{1}^{\prime} u+\psi_{1} u^{\prime}, y^{(\mathrm{iv})}=\psi_{1}^{(\mathrm{iv})} \widetilde{u}+4 \psi_{1}^{\prime \prime \prime} u+6 \psi_{1}^{\prime \prime} u^{\prime}+4 \psi_{1}^{\prime} u^{\prime \prime}+\psi_{1} u^{\prime \prime \prime}$. Substituting these expressions in (1.1) and taking into account the fact that $\psi_{1}$ satisfies (1.1), we obtain

$$
\psi_{1} u^{\prime \prime \prime}+4 \psi_{1}^{\prime} u^{\prime \prime}+6 \psi_{1}^{\prime \prime} u^{\prime}+4 \psi_{1}^{\prime \prime \prime} u+p\left(\psi_{1} u^{\prime}+2 \psi_{1}^{\prime} u\right)+p^{\prime} \psi_{1} u=0 .
$$

Dividing the two parts by $\psi_{1}$ and substituting the relations

$$
\frac{\psi_{1}^{\prime}}{\psi_{1}}=\sigma_{1}, \quad \frac{\psi_{1}^{\prime \prime}}{\psi_{1}}=\sigma_{1}^{\prime}+\sigma_{1}^{2}, \quad \frac{\psi_{1}^{\prime \prime \prime}}{\psi_{1}}=\sigma_{1}^{\prime \prime}+3 \sigma_{1}^{\prime} \sigma_{1}+\sigma_{1}^{3}, \quad \sigma_{\nu}=\frac{\psi_{\nu}^{\prime}}{\psi_{\nu}}, \quad \nu=1,2,
$$

we have

$$
u^{\prime \prime \prime}+4 \sigma_{1} u^{\prime \prime}+\left(6\left(\sigma_{1}^{\prime}+\sigma_{1}^{2}\right)+p\right) u^{\prime}+\left(4\left(\sigma_{1}^{\prime \prime}+3 \sigma_{1}^{\prime} \sigma_{1}+\sigma_{1}^{3}\right)+2 p \sigma_{1}+p^{\prime}\right) u=0 .
$$

Thus, the substitution $u=\left(\frac{y}{\psi_{1}}\right)^{\prime}$ lowers the order of equation (1.1) by one.

We repeat this procedure for (4.23), using the solution $\psi_{2}$ of equation (1.1). The asymptotic formulas (4.6) show that the function $g=\left(\frac{\psi_{2}}{\psi_{1}}\right)^{\prime}$ has no poles and does not vanish for $\lambda \in \mathscr{U}_{R}$. Moreover, $g$ satisfies (4.23). Let $u$ be a solution of (4.23). We show that the function $v=\left(\frac{u}{g}\right)^{\prime}$ satisfies an equation of the second order. We have $u=g \widetilde{v}$, where $\widetilde{v}=\int v(t) d t$. Then $u^{\prime}=g^{\prime} \widetilde{v}+g v, u^{\prime \prime}=g^{\prime \prime} \widetilde{v}+2 g^{\prime} v+g v^{\prime}, u^{\prime \prime \prime}=$ $g^{\prime \prime \prime} \widetilde{v}+3 g^{\prime \prime} v+3 g^{\prime} v^{\prime}+g v^{\prime \prime}$. Substituting these expressions in (4.23), we obtain

$$
g v^{\prime \prime}+3 g^{\prime} v^{\prime}+3 g^{\prime \prime} v+4 \sigma_{1}\left(g v^{\prime}+2 g^{\prime} v\right)+\left(6\left(\sigma_{1}^{\prime}+\sigma_{1}^{2}\right)+p\right) g v=0 .
$$

Denote $g_{1}=\frac{g^{\prime}}{g}$. Dividing the two parts of (4.24) by $g$ and using the relation $\frac{g^{\prime \prime}}{g}=g_{1}^{\prime}+g_{1}^{2}$, we have

(4.25) $v^{\prime \prime}+g_{2} v^{\prime}+\left(6\left(\sigma_{1}^{\prime}+\sigma_{1}^{2}\right)+8 \sigma_{1} g_{1}+3\left(g_{1}^{\prime}+g_{1}^{2}\right)+p\right) v=0$, where $g_{2}=4 \sigma_{1}+3 g_{1}$.

We have obtained an equation of the second order with an unknown function $v$.

Using a standard transformation, we reduce equation (4.25) to an equation that does not contain the term with the first derivative. We set $f= \pm v\left(\psi_{1}\right)^{2} g^{\frac{3}{2}}$, where we can take any fixed branch of the root $\sqrt{g}$ (recall that $g$ does not have poles and does not vanish in $\left.\mathscr{U}_{R}\right)$. Then $v=f h$, where $h= \pm\left(\psi_{1}\right)^{-2} g^{-\frac{3}{2}}$. Since $h^{\prime}=-\frac{h}{2} g_{2}$, we have $v^{\prime}=\frac{h}{2}\left(2 f^{\prime}-f g_{2}\right), v^{\prime \prime}=\frac{h}{4}\left(4 f^{\prime \prime}-4 f^{\prime} g_{2}-2 f g_{2}^{\prime}+f g_{2}^{2}\right)$. Substituting these relations in (4.25), we obtain (4.21), where

$$
V=4 \sigma_{1}^{\prime}+\frac{3}{2} g_{1}^{\prime}+2 \sigma_{1}^{2}+\frac{3}{4} g_{1}^{2}+2 \sigma_{1} g_{1}+p, \quad g_{1}=\frac{g^{\prime}}{g}, \quad g=\left(\frac{\psi_{2}}{\psi_{1}}\right)^{\prime} .
$$

Next we have $g=\frac{w_{12}}{\left(\psi_{1}\right)^{2}}$, which implies that

$$
g_{1}=\frac{g^{\prime}}{g}=\frac{w^{\prime}}{w}-2 \frac{\psi_{1}^{\prime}}{\psi_{1}}, \quad g_{1}^{\prime}=\frac{w^{\prime \prime}}{w}-\left(\frac{w^{\prime}}{w}\right)^{2}-2 \frac{\psi_{1}^{\prime \prime}}{\psi_{1}}+2\left(\frac{\psi_{1}^{\prime}}{\psi_{1}}\right)^{2} .
$$


On substituting these relations in (4.26), in view of the formulas $\sigma_{1}=\frac{\psi_{1}^{\prime}}{\psi_{1}}, \sigma_{1}^{\prime}=\frac{\psi_{1}^{\prime \prime}}{\psi_{1}}-$ $\left(\frac{\psi_{1}^{\prime}}{\psi_{1}}\right)^{2}$, we get

$$
V=\frac{3}{2} \frac{w^{\prime \prime}}{w}-\frac{3}{4}\left(\frac{w^{\prime}}{w}\right)^{2}+\frac{\psi_{1}^{\prime \prime}}{\psi_{1}}-\frac{w^{\prime}}{w} \frac{\psi_{1}^{\prime}}{\psi_{1}}+p
$$

which implies (4.22).

Note that $w^{\prime}=w_{02}$ and $w^{\prime \prime}=w_{12}+w_{03}$. Substituting these relations in (4.22) and using (4.18), we arrive at

$$
V=\frac{1}{2} \frac{w_{12}}{w_{01}}+\frac{3}{2} \frac{w_{03}}{w_{01}}-\frac{3}{4}\left(\frac{w_{02}}{w_{01}}\right)^{2}+p=\frac{1}{2} \frac{A_{12}}{A_{01}}+\frac{3}{2} \frac{A_{03}}{A_{01}}-\frac{3}{4}\left(\frac{A_{02}}{A_{01}}\right)^{2}+p .
$$

Then Lemma 4.3, ii) implies that the function $V(t, \cdot)$ is meromorphic in the domain $\mathscr{L}_{R}$ and is real on the interval $(-\infty,-R)$.

Remark. We believe (this has not been proved as yet) that a stronger assertion holds: every function $V(t, \cdot), t \in \mathbb{R}$, is analytic in the entire domain $\mathscr{L}_{R}$. If this is valid, then, using the method applied in 20 for proving the fact that the resonances with large modulus of the periodic third-order operator are real, one can prove that the resonances of $H$ with large modulus are real for arbitrary (and also smooth) coefficients $p, p^{\prime}$, $q \in L^{1}(0,1)$ of equation (1.1).

\section{$\S 5$. Proofs of Theorems 1.21 .3}

We shall need some results concerning the behavior of the Lyapunov function $\Delta(\lambda)$ in the interval of values $-1 \leq \Delta \leq 1$ (see Figure 4). Recall that $\Delta_{+}=\max \left\{\Delta_{1}, \Delta_{2}\right\}$ and $\Delta_{-}=\min \left\{\Delta_{1}, \Delta_{2}\right\}$ on any interval of the real axis on which $\rho>0$, i.e., $\operatorname{Im} \Delta=0$.

Lemma 5.1. Let $r$ be a zero of $\rho$ of multiplicity $m$, and let $\beta=\Delta_{1}(r)=\Delta_{2}(r) \in[-1,1]$. Then $m \leq 2$. Moreover, the following is valid.

i) Let $m=1$. If $\rho^{\prime}(r)>0$, then the $\Delta_{ \pm}$are real-analytic on the interval $(r, r+\delta)$ with some $\delta>0$ and $\Delta_{+}^{\prime}>0, \Delta_{-}^{\prime}<0$ on this interval. If $\rho^{\prime}(r)<0$, then the $\Delta_{ \pm}$are real-analytic on the interval $(r-\delta, r)$ with some $\delta>0$ and $\Delta_{+}^{\prime}<0, \Delta_{-}^{\prime}>0$ on this interval.

ii) If $m=2$, then $\rho^{\prime \prime}(r)>0$ and $\Delta_{1}$ and $\Delta_{2}$ are real-analytic on the interval $(r-\delta, r+\delta)$ with some $\delta>0$.

Proof. If $r$ is a zero of $\rho$ with multiplicity $m$, then $\rho(\lambda)=(\lambda-r)^{m} g(\lambda)$, where $g$ is an entire function real on $\mathbb{R}$, and $g(r) \neq 0$. Relations (1.4) yield

$$
\Delta(\lambda)=\beta+(\lambda-r)^{\frac{m}{2}} \sqrt{g(r)}+o\left(|\lambda-r|^{\frac{m}{2}}\right), \quad \lambda \in \mathbb{C}, \quad|\lambda-r| \rightarrow 0 .
$$

Assume for definiteness that $\beta \in(-1,1]$. The case of $\beta=-1$ is proved similarly. Consider the segment $[\beta-\delta, \beta]$ in the plane of $\Delta$, with some $\delta>0$ sufficiently small. If $m \geq 3$, then this segment has a preimage that does not lie on the real axis. Thus, $\Delta(\lambda) \in[\beta-\delta, \beta] \subset[-1,1]$ for a certain nonreal $\lambda$. By Theorem 1.1, iii), these $\lambda$ belong to the spectrum of $H$, which contradicts the selfadjointness of $H$. Therefore, $m \leq 2$.

i) We consider the case where $\rho^{\prime}(r)>0$. The case of $\rho^{\prime}(r)<0$ is treated similarly. If $\rho^{\prime}(r)>0$, then $\rho>0$ on the interval $u=(r, r+\delta)$ with some $\delta>0$. We have $\Delta_{ \pm}=T_{1} \pm|\sqrt{\rho}|$ on $u$ (see (1.4)), whence $\Delta_{ \pm}^{\prime}=T_{1}^{\prime} \pm \frac{\rho^{\prime}}{2|\sqrt{\rho}|}$. These relations imply that $\Delta_{+}^{\prime}>0$ and $\Delta_{-}^{\prime}<0$ on $u$.

ii) Let $r$ be a zero of $\rho$ with multiplicity $m=2$. Then $\rho(\lambda)=(\lambda-r)^{2} g(\lambda), g(r) \neq 0$, and $\rho^{\prime \prime}(r)=2 g(r)$. Assume that $g(r)<0$. The relation $\Delta=T_{1}+\sqrt{\rho}$ implies that $\Delta(\lambda)=\beta+i(\lambda-r) \sqrt{-g(r)}+o(|\lambda-r|),|\lambda-r| \rightarrow 0$. Under the mapping $\Delta$, the interval $(\beta-\varepsilon, \beta+\varepsilon)$ with some $\varepsilon>0$ has preimage orthogonal to the real axis $\lambda$. Since 

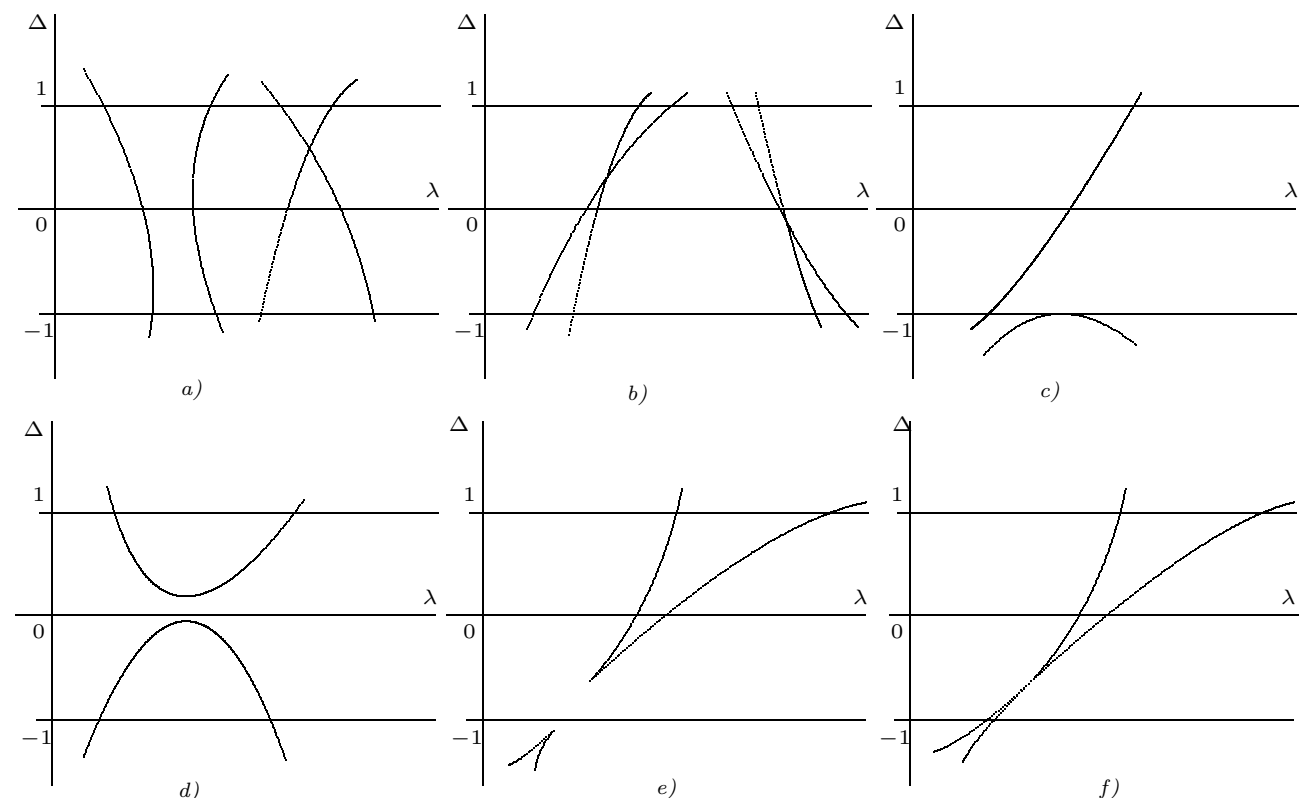

Figure 4. A possible (a, b) and an impossible (c, d, e, f) local behavior of the function $\Delta(\lambda)$ in the interval $-1 \leq \Delta \leq 1$.

$\beta \in[-1,1]$, we conclude that $\Delta(\lambda) \in[-1,1]$ for some nonreal $\lambda$, which contradicts the selfadjointness of $H$. Consequently, $g(r)>0$ and $\rho^{\prime \prime}(r)>0$. Then $r$ is not a branch point of the function $\Delta$ and $\Delta_{1}, \Delta_{2}$ are real-analytic in the interval $(r-\delta, r+\delta)$.

Remark. Repeating the arguments in the proof of Lemma 5.1] we derive that if $\Delta_{\nu}(\mu)=$ \pm 1 for some $(\nu, \mu) \in\{1,2\} \times \mathbb{R}$ and $\Delta_{\nu}^{\prime}(\mu)=0$, then $\Delta_{\nu}(\mu) \Delta_{\nu}^{\prime \prime}(\mu)<0$.

Recall that we have denoted by $\mathcal{R}$ the set of all real resonances and by $\mathcal{R}_{0}$ the subset of $\mathcal{R}$ that consists of "essential" resonances, which are endpoints of spectral bands. We briefly describe the behavior of the functions $\Delta_{1}$ and $\Delta_{2}$ in a neighborhood of a resonance $r$ that belongs to the set

$$
\mathcal{R}_{1}=\left\{r \in \mathcal{R} \backslash \mathcal{R}_{0}: \Delta_{1}(r)=\Delta_{2}(r) \in(-1,1)\right\} .
$$

The resonance $r \in \mathcal{R}_{1}$ has multiplicity 2 , and thus the functions $\Delta_{1}$ and $\Delta_{2}$ are realanalytic in a neighborhood of $r$. Moreover, $\Delta_{1}^{\prime} \Delta_{2}^{\prime}>0$ on the interval $(r-\delta, r+\delta)$ with some $\delta>0$ (Figure 4, b).

For $\alpha \in[-1,1]$, we consider the entire functions $D_{\alpha}(\lambda)=\left(\Delta_{1}(\lambda)-\alpha\right)\left(\Delta_{2}(\lambda)-\alpha\right)$. Note that $D_{ \pm 1}=D_{ \pm}, D_{\alpha}^{0}=(\cosh z-\alpha)(\cos z-\alpha)$.

Lemma 5.2. Let $\Delta_{\nu}\left(\mu_{0}\right)=\alpha_{0} \in(-1,1)$ for a branch $\nu= \pm$ and $\mu_{0} \in \mathbb{R} \backslash \mathcal{R}$. Then there exists a continuous, real, piecewise analytic function $\mu(\alpha), \alpha \in[-1,1]$, satisfying the following conditions:

a) $\mu(\alpha) \neq$ const, $D_{\alpha}(\mu(\alpha))=0$ for all $\alpha \in[-1,1], \mu\left(\alpha_{0}\right)=\mu_{0}$;

b) $\mu(1)=\eta_{1}$ is a periodic eigenvalue, $\mu(-1)=\eta_{2}$ is an antiperiodic eigenvalue;

c) inside the interval $S=\mu([-1,1])$, there are no periodic and antiperiodic eigenvalues, except possibly for $\eta_{1}$ and $\eta_{2}$;

d) each of the resonances that lies inside $S$ belongs to $\mathcal{R}_{1}$. 
Moreover, precisely one of the following three statements is valid:

1) $S=\left[\eta_{1}, \eta_{2}\right]$ or $S=\left[\eta_{2}, \eta_{1}\right], \Delta_{\nu}$ is continuous and monotone on $S$, and $\Delta_{\nu}(S)=$ $[-1,1]$

2) $S=S_{-} \cup S_{+}$, where $S_{+}=\left[r_{-}, \eta_{1}\right], S_{-}=\left[r_{-}, \eta_{2}\right], r_{-}$is a resonance from $\mathcal{R}_{0}$ such that $r_{-}<\eta_{-}=\min \left\{\eta_{1}, \eta_{2}\right\}$ and there are no resonances on the interval $\left(r_{-}, \eta_{-}\right)$. Next, $\Delta_{+}\left(S_{+}\right)=\left[\beta_{-}, 1\right], \Delta_{-}\left(S_{-}\right)=\left[-1, \beta_{-}\right]$, where $\beta_{-}=\Delta_{+}\left(r_{-}\right)=\Delta_{-}\left(r_{-}\right)$Moreover, the function $\Delta_{+}$is continuous and monotone increasing on $S_{+}$, whereas $\Delta_{-}$is continuous and monotone decreasing on $S_{-}$.

3) $S=S_{-} \cup S_{+}$, where $S_{+}=\left[\eta_{1}, r_{+}\right], S_{-}=\left[\eta_{2}, r_{+}\right], r_{+}$is a resonance from $\mathcal{R}_{0}$ such that $r_{+}>\eta_{+}=\max \left\{\eta_{1}, \eta_{2}\right\}$, and there are no resonances on the interval $\left(\eta_{+}, r_{+}\right)$. Next, $\Delta_{+}\left(S_{+}\right)=\left[\beta_{+}, 1\right], \Delta_{-}\left(S_{-}\right)=\left[-1, \beta_{+}\right]$, where $\beta_{+}=\Delta_{+}\left(r_{+}\right)=\Delta_{-}\left(r_{+}\right)$. Moreover, the function $\Delta_{+}$is continuous and monotone decreasing on $S_{+}$, whereas $\Delta_{-}$is continuous and monotone increasing on $S_{-}$.

Proof. Since $\mu_{0} \notin \mathcal{R}$, the function $\Delta_{\nu}$ is real-analytic in a neighborhood of $\mu_{0}$. Assume for definiteness that $\nu=+$ and $\Delta_{+}^{\prime}\left(\mu_{0}\right)>0$; the proofs for the other cases are similar.

Using Theorem 1.1, ii) and properties of the functions $\Delta_{1}$ and $\Delta_{2}$ in a neighborhood of a resonance lying in $\mathcal{R}_{1}$, we conclude that the function $\Delta_{+}$is real-analytic on a set of the form $\left(\alpha_{1}, \alpha_{2}\right) \backslash \mathcal{R}_{1}$ that contains the point $\mu_{0}$, is continuous, and increases monotonically on $\left(\alpha_{1}, \alpha_{2}\right)$. Here $\alpha_{1}$ is a resonance from $\mathcal{R}_{0}$ or an antiperiodic eigenvalue, and $\alpha_{2}$ is a periodic eigenvalue. By the inverse function theorem, there exists a continuous, real, piecewise analytic function $\mu(\alpha), \alpha \in\left[\alpha_{1}, \alpha_{2}\right]$, such that $\Delta_{+}(\mu(\alpha))=\alpha$ for all $\alpha \in$ $\left[\alpha_{1}, \alpha_{2}\right]$, and $\mu(\alpha)$ increases monotonically on this interval. If $\alpha_{1}$ is an antiperiodic eigenvalue, then statement 1) with $\eta_{1}=\alpha_{2}$ and $\eta_{2}=\alpha_{1}$ is valid, and the lemma is proved for this case.

Suppose $\alpha_{1}$ is a resonance from $\mathcal{R}_{0}$. Let $\eta_{1}$ and $\eta_{2}$ be the periodic and antiperiodic eigenvalues, respectively, closest to $\alpha_{1}$ and lying on the right of $\alpha_{1}$. We show that statement 2) is valid, where $r_{-}=\alpha_{1}$ and $\eta_{1}=\alpha_{2}$. We assume for definiteness that $\eta_{1} \leq \eta_{2}$, i.e., $\eta_{-}=\eta_{1}$; the case where $\eta_{1}>\eta_{2}$ is considered similarly.

We show that the interval $\left(\alpha_{1}, \eta_{1}\right]$ contains no resonances. Suppose this interval contains resonances, and let $\widetilde{r}_{0}$ be the smallest of them. Then $\rho>0$ on $\left(\alpha_{1}, \widetilde{r}_{0}\right)$, the $\Delta_{ \pm}$are real-analytic on this interval, and $\Delta_{+}=\Delta_{-}$at the endpoints of it. Since $\alpha_{1} \in \mathcal{R}_{0}$, we have $\Delta_{+}^{\prime}>0, \Delta_{-}^{\prime}<0$ on $\left(\alpha_{1}, \alpha_{1}+\delta\right)$ with some $\delta>0$. By Theorem 1.1, ii $), \Delta_{+}^{\prime}>0$ on $\left(\alpha_{1}, c_{1}\right), \Delta_{-}^{\prime}<0$ on $\left(\alpha_{1}, c_{2}\right)$, where $c_{1}, c_{2} \in\left(\alpha_{1}, \widetilde{r}_{0}\right)$ are a periodic and an antiperiodic eigenvalue, respectively. This contradicts the condition that $\eta_{1}$ and $\eta_{2}$ are the periodic and antiperiodic eigenvalues closest to $\alpha_{1}$ from the right. Consequently, the interval $\left(\alpha_{1}, \eta_{1}\right]$ contains no resonances, whence, by the definition of $r_{-}$, we obtain $\alpha_{1}=r_{-}$.

Since the interval $\left(r_{-}, \eta_{1}\right]$ does not contain resonances, Theorem [1.1] ii) implies that the functions $\Delta_{ \pm}$are real-analytic on $\left(r_{-}, \eta_{1}\right), \Delta_{+}^{\prime}>0$ and $\Delta_{-}^{\prime}<0$ on this interval, and $\Delta_{+}\left(S_{+}\right)=\left[\beta_{-}, 1\right]$. By the inverse function theorem, there exists a real, continuous, piecewise analytic function $\mu^{+}(\alpha), \alpha \in\left[\beta_{-}, 1\right]$, such that $\mu^{+}\left(\beta_{-}\right)=r_{-}$and $\Delta_{+}\left(\mu^{+}(\alpha)\right)=$ $\alpha$ for all $\alpha \in\left[\beta_{-}, 1\right]$.

We show that if there are resonances on the interval $\left(\eta_{1}, \eta_{2}\right)$, then they belong to $\mathcal{R}_{1}$. Note that $\Delta_{-}\left(\eta_{1}\right) \in(-1,1)$ and, since $\eta_{1}$ is not a resonance, it follows that $\Delta_{-}$is realanalytic in a neighborhood of $\eta_{1}$ and $\Delta_{-}^{\prime}\left(\eta_{1}\right)<0$. Let $\widetilde{r}_{1}$ be the smallest resonance from $\mathcal{R}_{0}$ that lies in $\left(\eta_{1}, \eta_{2}\right)$. Then all the resonances in the interval $\left(\eta_{1}, \widetilde{r}_{1}\right)$ belong to $\mathcal{R}_{1}$.

Using Theorem[1.1, ii) and properties of the functions $\Delta_{1}$ and $\Delta_{2}$ in a neighborhood of a resonance from $\mathcal{R}_{1}$, we conclude that $\Delta_{-}^{\prime}<0$ on $\left(\eta_{1}, \widetilde{r}_{1}\right) \backslash \mathcal{R}_{1}$. On the other hand, since $\widetilde{r}_{1} \in \mathcal{R}_{0}$, we have $\Delta_{+}^{\prime}<0, \Delta_{-}^{\prime}>0$ on $\left(\widetilde{r}_{1}-\delta_{1}, \widetilde{r}_{1}\right)$ with some $\delta_{1}>0$, a contradiction. Consequently, the interval $\left(\eta_{1}, \eta_{2}\right)$ contains no resonances from $\mathcal{R}_{0}$. 
Thus, the interval $\left(r_{-}, \eta_{2}\right)$ contains no resonances from $\mathcal{R}_{0}$. Using Theorem 1.1, ii) and properties of the functions $\Delta_{1}$ and $\Delta_{2}$ in a neighborhood of a resonance $r$ from $\mathcal{R}_{1}$, we conclude that $\Delta_{-}$is a continuous monotone decreasing function on $\left(r_{-}, \eta_{2}\right)$. Then $\Delta_{-}\left(S_{-}\right)=\left[-1, \beta_{-}\right]$, and by the inverse function theorem, there exists a real, continuous, piecewise analytic function $\mu^{-}(\alpha), \alpha \in\left[-1, \beta_{-}\right]$, such that $\mu^{-}\left(\beta_{-}\right)=r_{-}$and $\Delta_{-}\left(\mu^{-}(\alpha)\right)=\alpha$ for all $\alpha \in\left[-1, \beta_{-}\right]$.

Putting

$$
\mu(\alpha)=\left\{\begin{array}{lll}
\mu^{-}(\alpha) & \text { if } & \alpha \in\left[-1, \beta_{-}\right], \\
\mu^{+}(\alpha) & \text { if } & \alpha \in\left[\beta_{-}, 1\right],
\end{array}\right.
$$

we deduce statement 2) of the lemma in the case under consideration.

Proof of Theorem 1.2. i) Every function $D_{\alpha}^{0}, \alpha \in(-1,1)$, has simple zeros at the points $\arccos ^{4} \alpha,(2 \pi n \pm \arccos \alpha)^{4}, n \geq 1, \arccos \alpha \in(0, \pi)$, and has no other zeros. Repeating arguments adduced in the proof of Lemma 3.4, we see that for each integer $N>n_{0}$ with a certain $n_{0} \geq 1$, every function $D_{\alpha}, \alpha \in(-1,1)$, has precisely $N$ zeros (counted with multiplicity) in the domain $\left\{|\lambda|<(\pi N)^{4}\right\}$ and for each $n>N$ precisely one simple zero in every domain $\left\{\left|\lambda^{1 / 4}-(2 \pi n \pm \arccos \alpha)\right|<1-|\alpha|\right\}$. This function has no other zeros.

By Theorem 1.1 iii), all the zeros of $D_{\alpha}$ are points of the spectrum, and thus they are real. We enumerate the zeros $\mu_{n}(\alpha), n \geq 1$, of the function $D_{\alpha}, \alpha \in[-1,1]$, counted with multiplicity, in such a way that $\mu_{1}(\alpha) \leq \mu_{2}(\alpha) \leq \cdots$. By the implicit function theorem, every zero $\mu_{n}(\alpha)$ of $D_{\alpha}$ depends on $\alpha \in[-1,1]$ continuously.

We show that for all $\alpha \in(-1,1)$ except for several values $\alpha_{1}, \alpha_{2}, \ldots, \alpha_{k}, k \geq 0$, all zeros $\mu_{n}(\alpha)$ of $D_{\alpha}$ are simple, and the functions $\mu_{n}(\alpha)$ are real-analytic on the set $A=(-1,1) \backslash\left\{\alpha_{1}, \alpha_{2}, \ldots, \alpha_{k}\right\}$. Indeed, suppose $\mu=\mu_{n}\left(\alpha_{0}\right)$ is a zero of $D_{\alpha_{0}}, \alpha_{0} \in(-1,1)$, and $\mu$ is not a resonance. Then a) $\mu$ is a zero of $\Delta_{\nu}-\alpha_{0}$ with any one $\nu \in\{1,2\}$; b) by Theorem [1.1, ii), $\Delta_{\nu}^{\prime}(\mu) \neq 0$. This implies that $\partial_{\lambda} D_{\alpha_{0}}(\mu) \neq 0$ and, by the implicit function theorem, $\mu$ is a simple zero of $D_{\alpha_{0}}$ and depends on $\alpha$ analytically in a neighborhood of $\alpha_{0}$. From the asymptotic relation (3.17), it follows that there are at most finitely many resonances on the positive semiaxis. Therefore, for all $\alpha \in(-1,1)$ except for a finite number of them, there are no resonances among the zeros of the function $D_{\alpha}$, which implies that for all $\alpha \in A$, all the zeros $\mu_{n}(\alpha)$ of the function $D_{\alpha}$ are simple and the functions $\mu_{n}(\alpha)$ are real-analytic on the set $A$.

Since, by Lemma 3.4, the number of zeros of $D_{\alpha}$ (counted with multiplicity) for $\alpha$ close to 1 coincides with the number of zeros of the function $D_{+}=D_{1}$, and inequalities (1.7) are valid, we conclude that $\mu_{2 n-1}(1)=\lambda_{2 n-2}^{+}$and $\mu_{2 n}(1)=\lambda_{2 n}^{-}, n \geq 1$. Thus, for every $n \geq 1$ there exists a unique piecewise analytic function $\mu_{n}(\alpha), \alpha \in[-1,1]$, such that $D_{\alpha}\left(\mu_{n}(\alpha)\right)=0, \mu_{2 n-1}(1)=\lambda_{2 n-2}^{+}, \mu_{2 n}(1)=\lambda_{2 n}^{-}$, and $\mu_{1}(\alpha)<\mu_{2}(\alpha)<\cdots$ for all $\alpha \in A$. Moreover, $\mu_{2 n-1}(-1)=\lambda_{2 n-1}^{-}$and $\mu_{2 n}(-1)=\lambda_{2 n-1}^{+}$for all $n \geq 1$.

By Lemma 5.2, for every function $\mu_{n}(\alpha)$, precisely one of the statements 1), 2), 3) holds true. This implies that precisely one of the cases I, II, III of the theorem may occur.

Assume that $\sigma_{1}=\left[\lambda_{1}, r_{1}\right]$ satisfies condition III, where $\lambda_{1}=\min \left\{\lambda_{0}^{+}, \lambda_{1}^{-}\right\}, \lambda_{0}^{+}$and $\lambda_{1}^{-}$are simple periodic and antiperiodic eigenvalues, and $r_{1} \in \mathcal{R}_{0}$. Let, for definiteness, $\lambda_{0}^{+} \leq \lambda_{1}^{-}$, i.e., $\lambda_{1}=\lambda_{0}^{+}$; the opposite case is considered similarly. Then $\rho>0$ on the interval $\left[\lambda_{1}^{-}, r_{1}\right)$. We show that $\rho>0$ on the interval $\left(-\infty, \lambda_{1}^{-}\right]$. Assume that $\tilde{r}$ is the greatest resonance on this interval. Then $\rho>0$ on $\left(\tilde{r}, \lambda_{1}^{-}\right)$and $\Delta_{+}(\lambda)>\Delta_{+}\left(\lambda_{1}^{-}\right)>-1$ for all $\lambda \in\left[\widetilde{r}, \lambda_{1}^{-}\right)$. Since $\Delta_{+}(\widetilde{r})=\Delta_{-}(\widetilde{r})>-1$, there exists at least one antiperiodic eigenvalue on the interval $\left(\widetilde{r}, \lambda_{1}^{-}\right)$. Since $\lambda_{1}^{-}$is the least antiperiodic eigenvalue, we obtain a contradiction. Consequently, there are no resonances on the interval $\left(-\infty, \lambda_{1}^{-}\right]$ 
and $\rho>0$ on this interval, which contradicts the asymptotic relation (3.17). Therefore, $\sigma_{1}$ cannot satisfy condition III.

From the above, it follows that $\left[\lambda_{n-1}^{+}, \lambda_{n}^{-}\right]\left(\right.$or $\left.\left[\lambda_{n}^{-}, \lambda_{n-1}^{+}\right]\right)$is included in $\sigma_{n},\left[\lambda_{n}^{+}, \lambda_{n+1}^{-}\right]$ (or $\left.\left[\lambda_{n+1}^{-}, \lambda_{n}^{+}\right]\right)$is included in $\sigma_{n+1}$ and $\left[\lambda_{n+1}^{+}, \lambda_{n+2}^{-}\right]\left(\right.$or $\left.\left[\lambda_{n+2}^{-}, \lambda_{n+1}^{+}\right]\right)$is included in $\sigma_{n+2}$. The inequalities $\lambda_{n-1}^{+} \leq \lambda_{n+1}^{-} \leq \lambda_{n+1}^{+}, \lambda_{n}^{-} \leq \lambda_{n}^{+} \leq \lambda_{n+2}^{-}$imply that if $\sigma_{n} \cap \sigma_{n+2} \neq \varnothing$, then $\widetilde{\sigma}=\sigma_{n} \cap \sigma_{n+1} \cap \sigma_{n+2} \neq \varnothing$. Then the function $\Delta$ takes at least three distinct values at each point $\lambda \in \widetilde{\sigma}$, which contradicts the fact that the function $\Delta$ is two-sheeted. We obtain (1.12).

ii) Let $r$ be a resonance lying inside a band $\sigma_{n}$ with some $n \geq 1$. Then, by Theorem [1.2 i), $r$ has multiplicity 2 and does not belong to $\mathcal{R}_{0}$. Consequently, the functions $\Delta_{1}$ and $\Delta_{2}$ are real-analytic in a neighborhood of $r, \Delta_{1}(r)=\Delta_{2}(r) \in(-1,1)$, and $\Delta_{1}^{\prime}(r)$ and $\Delta_{2}^{\prime}(r)$ do not equal zero and have one and the same sign. This yields (1.13).

iii) Since $r \in \sigma(H)$, we have $r \in \sigma_{n}$ for some $n \geq 1$. On the other hand, by statement 1) of the theorem that we are proving, the interior of the interval $\sigma_{n}$ cannot contain resonances from $\mathcal{R}_{0}$. Therefore, $r$ is an endpoint of $\sigma_{n}$.

iv) If $\lambda$ belongs to the set $\mathfrak{S}_{4}$ defined by (1.14), then $\Delta(\lambda)$ takes two different values in $[-1,1]$. Then, by Theorem [1.1, iii), the spectrum has multiplicity 4 at the point $\lambda$. If $\lambda \in \mathfrak{S}_{2}$, then $\Delta_{\nu}(\lambda) \in[-1,1]$ for only one $\nu=1,2$. Therefore, the spectrum in $\mathfrak{S}_{2}$ has multiplicity 2 .

Proof of Theorem 1.3, i) The asymptotic relation (3.17) implies that $\rho>0$ on $[R,+\infty$ ) for some $R \in \mathbb{R}$, i.e., $\Delta$ is real on this interval. Moreover, the asymptotic formulas (1.6) show that $\Delta_{1} \notin[-1,1]$ on $[R,+\infty)$ for some $R \in \mathbb{R}$. Then, using the asymptotic formulas (1.6) for $\Delta_{2}$ and Theorem 1.1, ii), we conclude that every band $\sigma_{n}, n \geq n_{0}$, coincides with $\left[\lambda_{n-1}^{+}, \lambda_{n}^{-}\right]$, the spectrum in it has multiplicity 2 , and the intervals $\left(\lambda_{n}^{-}, \lambda_{n}^{+}\right)$are gaps.

We prove (1.15). By Lemma 3.3, $r_{n}^{ \pm} \in \mathcal{D}_{n}$ for all $n \geq 1$ sufficiently large. Let $\lambda$ be $r_{n}^{+}$or $r_{n}^{-}$, and let $\operatorname{Im} \lambda \geq 0$; the proof for the case of $\operatorname{Im} \lambda<0$ is similar. Then $z=\lambda^{1 / 4}=(1+i)(\pi n+\delta)$, where $\delta=\delta_{n}$ satisfies the inequality $|\delta|<\frac{\pi}{4}$ for all $n$ sufficiently large. The asymptotic formulas (3.18) yield

$$
0=\rho(\lambda)=\frac{e^{2 \pi n+2 \delta}}{16}\left(\left(\phi_{00}(\lambda) e^{i \delta}-\phi_{11}(\lambda) e^{-i \delta}\right)^{2}+4 \phi_{01}(\lambda) \phi_{10}(\lambda)+O\left(e^{-2 \pi n}\right)\right)
$$

as $n \rightarrow+\infty$. Using (3.7), we have $\phi_{j j}(\lambda)=1+O\left(n^{-1}\right), \phi_{k j}(\lambda)=O\left(n^{-2}\right), k \neq j$. Then (5.1) implies that $\sin \delta=O\left(n^{-1}\right)$, whence $\delta=O\left(n^{-1}\right)$. Again with the help of (3.7), we obtain

$$
\phi_{00}(\lambda)=e^{-\frac{(1-i) \xi}{2} \hat{p}_{0}}+O\left(\xi^{3}\right), \quad \phi_{11}(\lambda)=e^{-\frac{(1+i) \xi}{2} \hat{p}_{0}}+O\left(\xi^{3}\right), \quad \xi=\frac{1}{4 \pi n},
$$

as $n \rightarrow+\infty$. Substituting these asymptotic formulas and (3.10) in (5.1), we get

$$
0=\rho(\lambda)=\frac{e^{2 \pi n+2 \delta-\xi \hat{p}_{0}}}{4}\left(-\sin ^{2}\left(\delta+\frac{\xi \widehat{p}_{0}}{2}\right)+2\left|\widehat{p_{n}^{\prime}}\right|^{2} \xi^{4}+O\left(\xi^{4}\right)\right),
$$

whence $\delta=-\frac{\xi \hat{p}_{0}}{2}+\widetilde{\delta}, \widetilde{\delta}=O\left(\xi^{2}\right)$. Substituting these asymptotic formulas in (5.2), we have $\sin ^{2} \widetilde{\delta}-2 \xi^{4}\left|\widehat{p_{n}^{\prime}}\right|^{2}=O\left(\xi^{6}\right)$, which yields $\widetilde{\delta}= \pm \sqrt{2} \xi^{2}\left|\widehat{p_{n}^{\prime}}\right|+O\left(\xi^{3}\right)$ and

$$
\left(r_{n}^{ \pm}\right)^{\frac{1}{4}}=(1+i)\left(\pi n-\frac{\xi \hat{p}_{0}}{2}+\tilde{\delta}\right)=(1+i)\left(\pi n-\frac{\xi \widehat{p}_{0}}{2} \mp \sqrt{2} \xi^{2}\left|\widehat{p_{n}^{\prime}}\right|+O\left(\xi^{3}\right)\right),
$$

whence 1.15 follows.

Now we prove (1.16) for $\lambda=\lambda_{2 n}^{ \pm}$. The proof for $\lambda_{2 n-1}^{ \pm}$is similar. Recall that $\lambda=\lambda_{2 n}^{ \pm}$is a periodic eigenvalue, and it satisfies the relation $\operatorname{det}\left(M(\lambda)-I_{4}\right)=0$. Relation (3.5) 
implies that $0=\operatorname{det}\left(\Phi(\lambda) e^{z \Omega(\lambda)}-I_{4}\right)=\operatorname{det}\left(\Phi(\lambda)-e^{-z \Omega(\lambda)}\right), z=\lambda^{\frac{1}{4}}$ (recall that $\Omega(\lambda)=$ $\left.(1,-i, i,-1), \lambda \in \overline{\mathbb{C}_{+}}\right)$. By Lemma 3.4 $z=2 \pi n+\varepsilon$, where $\varepsilon=\varepsilon_{2 n}^{ \pm}$satisfies the inequality $|\varepsilon|<\frac{\pi}{2}$ for all $n$ sufficiently large. Then

$$
\Phi-e^{-z \Omega}=\left(\begin{array}{cccc}
\phi_{00}-e^{-2 \pi n-\varepsilon} & \phi_{01} & \phi_{02} & \phi_{03} \\
\phi_{10} & \phi_{11}-e^{i \varepsilon} & \phi_{12} & \phi_{13} \\
\phi_{20} & \phi_{21} & \phi_{22}-e^{-i \varepsilon} & \phi_{23} \\
\phi_{30} & \phi_{31} & \phi_{32} & \phi_{33}-e^{2 \pi n+\varepsilon}
\end{array}\right)
$$

throughout this proof, we write $\Phi=\Phi(\lambda), \phi_{k j}=\phi_{k j}(\lambda), \ldots$ Then

$$
\operatorname{det}\left(\Phi-e^{-z \Omega}\right)=e^{2 \pi n} \operatorname{det}\left(\begin{array}{cccc}
\phi_{00}-e^{-2 \pi n-\varepsilon} & \phi_{01} & \phi_{02} & \phi_{03} \\
\phi_{10} & \phi_{11}-e^{i \varepsilon} & \phi_{12} & \phi_{13} \\
\phi_{20} & \phi_{21} & \phi_{22}-e^{-i \varepsilon} & \phi_{23} \\
e^{-2 \pi n} \phi_{30} & e^{-2 \pi n} \phi_{31} & e^{-2 \pi n} \phi_{32} & e^{-2 \pi n} \phi_{33}-e^{\varepsilon}
\end{array}\right) .
$$

Using the first inequality in (3.6), we get

$\operatorname{det}\left(\Phi-e^{-z \Omega}\right)=-e^{2 \pi n+\varepsilon}\left(\operatorname{det}\left(\begin{array}{ccc}\phi_{00}-e^{-2 \pi n-\varepsilon} & \phi_{01} & \phi_{02} \\ \phi_{10} & \phi_{11}-e^{i \varepsilon} & \phi_{12} \\ \phi_{20} & \phi_{21} & \phi_{22}-e^{-i \varepsilon}\end{array}\right)+O\left(e^{-2 \pi n}\right)\right)$,

which yields

$$
0=\operatorname{det}\left(\Phi-e^{-z \Omega}\right)=-\phi_{00} e^{2 \pi n+\varepsilon}\left(F_{0}+F_{1}\right),
$$

where

$$
\begin{aligned}
& F_{0}=\operatorname{det}\left(\begin{array}{cc}
\phi_{11}-e^{i \varepsilon} & \phi_{12} \\
\phi_{21} & \phi_{22}-e^{-i \varepsilon}
\end{array}\right), \\
& F_{1}=-\frac{\phi_{01}}{\phi_{00}} \operatorname{det}\left(\begin{array}{cc}
\phi_{10} & \phi_{12} \\
\phi_{20} & \phi_{22}-e^{-i \varepsilon}
\end{array}\right)+\frac{\phi_{02}}{\phi_{00}} \operatorname{det}\left(\begin{array}{cc}
\phi_{10} & \phi_{11}-e^{i \varepsilon} \\
\phi_{20} & \phi_{21}
\end{array}\right)+O\left(e^{-2 \pi n}\right) .
\end{aligned}
$$

The asymptotic formulas (3.7) imply that

$$
F_{0}=\operatorname{det}\left(\begin{array}{cc}
e^{-\frac{i \hat{p}_{0}}{4 z}}-e^{i \varepsilon}+O\left(n^{-3}\right) & O\left(n^{-2}\right) \\
O\left(n^{-2}\right) & e^{\frac{i \hat{p}_{0}}{4 z}}-e^{-i \varepsilon}+O\left(n^{-3}\right)
\end{array}\right), \quad F_{1}=O\left(n^{-4}\right),
$$

$\lambda=\lambda_{2 n}^{ \pm}$, whence $F_{0}=2-2 \cos \left(\varepsilon+\frac{\hat{p}_{0}}{4 z}\right)+O\left(n^{-3}\right)$. From the relation $F_{0}+F_{1}=0$ it follows that $\varepsilon=-\frac{\xi \hat{p}_{0}}{2}+\widetilde{\varepsilon}, \widetilde{\varepsilon}=O\left(\xi^{\frac{3}{2}}\right), \xi=\frac{1}{4 \pi n}$. Moreover, $e^{-\frac{i \hat{p}_{0}}{4 z}}-e^{i \varepsilon}=O\left(\xi^{\frac{3}{2}}\right)$ and $e^{\frac{i \hat{p}_{0}}{4 z}}-e^{-i \varepsilon}=O\left(\xi^{\frac{3}{2}}\right)$. We obtain $F_{0}=2-2 \cos \left(\varepsilon+\frac{\hat{p}_{0}}{4 z}\right)+O\left(\xi^{4}\right)$, and thus $\widetilde{\varepsilon}=O\left(n^{-2}\right)$.

The asymptotic formulas (3.7) imply that $\phi_{11}(\lambda)-e^{i \varepsilon}=O\left(\xi^{2}\right), \phi_{22}(\lambda)-e^{-i \varepsilon}=O\left(\xi^{2}\right)$. Substituting (3.7) and (3.9) in (5.3), we arrive at $F_{1}=O\left(n^{-6}\right)$ and

$$
\begin{aligned}
& F_{0}=\operatorname{det}\left(\begin{array}{cc}
e^{-\frac{i \hat{p}_{0}}{4 z}}-e^{i \varepsilon}+O\left(\xi^{3}\right) & -\frac{1}{2} \xi^{2} \widehat{\widehat{p_{2 n}^{\prime}}}+O\left(\xi^{3}\right) \\
-\frac{1}{2} \xi^{2} \widehat{p_{2 n}^{\prime}}+O\left(\xi^{3}\right) & e^{i \hat{p}_{0}}-e^{-i \varepsilon}+O\left(\xi^{3}\right)
\end{array}\right) \\
& =\operatorname{det}\left(\begin{array}{cc}
e^{-\frac{i \xi \hat{p}_{0}}{2}}\left(1-e^{i \tilde{\varepsilon}}\right)+O\left(\xi^{3}\right) & -\frac{1}{2} \xi^{2} \widehat{\widehat{p_{2 n}^{\prime}}}+O\left(\xi^{3}\right) \\
-\frac{1}{2} \xi^{2} \widehat{p_{2 n}^{\prime}}+O\left(\xi^{3}\right) & e^{\frac{i \hat{p}_{0}}{2}}\left(1-e^{-i \widetilde{\varepsilon}}\right)+O\left(\xi^{3}\right)
\end{array}\right) \\
& =2-2 \cos \widetilde{\varepsilon}-\frac{\xi^{4}}{4}\left|\widehat{p_{2 n}^{\prime}}\right|^{2}+\left(\sin \frac{\xi \widehat{p}_{0}}{2}+\sin \left(\widetilde{\varepsilon}-\frac{\xi \widehat{p}_{0}}{2}\right)\right) O\left(\xi^{3}\right)+O\left(\xi^{5}\right) \\
& =\widetilde{\varepsilon}^{2}-\frac{\xi^{4}}{4}\left|\widehat{p_{2 n}^{\prime}}\right|^{2}+\widetilde{\varepsilon} O\left(\xi^{3}\right)+O\left(\xi^{5}\right) \text {. }
\end{aligned}
$$


The relation $F_{0}+F_{1}=0$ yields $\widetilde{\varepsilon}= \pm \frac{\xi^{2}}{2}\left|\widehat{p_{2 n}^{\prime}}\right|+O\left(\xi^{3}\right)$. Then

$$
\left(\lambda_{2 n}^{ \pm}\right)^{\frac{1}{4}}=2 \pi n-\frac{\xi \widehat{p}_{0}}{2} \pm \frac{\xi^{2}}{2}\left|\widehat{p_{2 n}^{\prime}}\right|+O\left(\xi^{3}\right),
$$

whence (1.16) follows for $\lambda_{2 n}^{ \pm}$.

The asymptotic behavior (1.16) and the estimates $\left|\widehat{p_{n}^{\prime}}\right| \geq \frac{1}{n^{\alpha}}$ imply the asymptotic relation $\left|\gamma_{n}\right|=\lambda_{n}^{+}-\lambda_{n}^{-}=\pi n\left|\widehat{p_{n}^{\prime}}\right|+O(1)$ and the estimates $\left|\gamma_{n}\right| \geq \pi n^{1-\alpha}\left(1+O\left(n^{\alpha-1}\right)\right)$ as $n \rightarrow \infty$. It follows that there exist infinitely many gaps $\gamma_{n} \neq \varnothing$, and $\left|\gamma_{n}\right| \rightarrow \infty$ as $n \rightarrow \infty$.

Now we prove the fact that in this case, the resonances large in modulus are real. Suppose the contrary; i.e., suppose a resonance $r_{n}^{ \pm}$with large $n$ is nonreal. Then $r_{n}^{-}=\overline{r_{n}^{+}}$, which contradicts the asymptotic behavior (1.15). Consequently, $r_{n}^{ \pm} \in \mathbb{R}$. Moreover, the asymptotic behavior (1.15) yields $\left|r_{n}^{+}-r_{n}^{-}\right|=2 \sqrt{2} \pi n\left|\widehat{p_{n}^{\prime}}\right|+O(1)$ as $n \rightarrow \infty$, whence $\left|r_{n}^{+}-r_{n}^{-}\right| \rightarrow \infty$ as $n \rightarrow \infty$.

ii) With the help of relations (3.14), (3.15), and the asymptotic behavior (1.15), (1.16), repeating the standard arguments from 1 based on the Hadamard factorization of the entire functions $D_{ \pm}$and $\rho$, we obtain the required statements.

\section{$\S 6$. The spectrum of $H$ with SMall Coefficients}

Proof of Theorem 1.4. The arguments adduced in the proof of Lemma 2.1 show that the functions $T_{\nu}(\lambda, \varepsilon p), \nu=1,2$, are entire functions of the variables $\lambda$ and $\varepsilon$ for any fixed $p$ in the set considered. Then $T_{\nu}^{\varepsilon}(\lambda)=T_{\nu}(\lambda, \varepsilon p), \rho^{\varepsilon}(\lambda)=\rho(\lambda, \varepsilon p)$, and $D_{ \pm}^{\varepsilon}(\lambda)=D_{ \pm}(\lambda, \varepsilon p)$ are entire functions of the variable $\varepsilon$. Using the relation $\hat{p}_{0}=0$, we can achieve $p(0)=0$ by shifting the argument $t$ in equation (1.1) (recall that such a shift of the argument does not change the Lyapunov function and the spectrum of the operator $H$ ). From estimate (2.4), we have

$$
T_{\nu}^{\varepsilon}(\lambda)=T_{\nu}^{0}(\lambda)+\varepsilon^{2} \eta_{\nu}(\lambda)+O\left(\varepsilon^{3}\right), \quad \varepsilon \rightarrow 0,
$$

uniformly on every bounded subset in $\mathbb{C}$. Substituting (6.1) in (1.3), we get

$$
\rho^{\varepsilon}(\lambda)=\rho^{0}(\lambda)+\varepsilon^{2} \tilde{\rho}(\lambda, \varepsilon), \quad \tilde{\rho}(\lambda, \varepsilon)=\frac{\eta_{2}}{2}-2 T_{1}^{0}(\lambda) \eta_{1}(\lambda)+O(\varepsilon), \quad \varepsilon \rightarrow 0,
$$

uniformly on every bounded subset in $\mathbb{C}$. The function $\rho^{0}$ has the simple zero $\lambda=0$, and $\widetilde{\rho}$ is analytic at the point $(\lambda, \varepsilon)=(0,0)$. Applying the implicit function theorem to $\rho^{\varepsilon}=\rho^{0}+\varepsilon^{2} \widetilde{\rho}$, where $\left.\partial_{\lambda} \rho^{\varepsilon}\right|_{\lambda=\varepsilon=0} \neq 0$, we find a unique solution $r_{0}^{-}(\varepsilon)$ of the equation $\rho^{\varepsilon}(\lambda)=0$ in the domain $|\varepsilon|<\varepsilon_{1}$ with some $\varepsilon_{1}>0$ that satisfies the condition $r_{0}^{-}(0)=0$.

Substituting (6.1) in (3.15), we have

$$
D_{+}^{\varepsilon}(\lambda)=D_{+}^{0}(\lambda)+\varepsilon^{2} \widetilde{D}_{+}(\lambda, \varepsilon), \quad \widetilde{D}_{+}(\lambda, \varepsilon)=2\left(2 T_{1}^{0}(\lambda)-1\right) \eta_{1}(\lambda)-\frac{\eta_{2}(\lambda)}{2}+O(\varepsilon)
$$

as $\varepsilon \rightarrow 0$ uniformly on any bounded subset in $\mathbb{C}$. The function $D_{+}^{0}$ has the simple zero $\lambda=0$, and $\widetilde{D}_{+}$is analytic at the point $(\lambda, \varepsilon)=(0,0)$. Applying the implicit function theorem to $D_{+}^{\varepsilon}=D_{+}^{0}+\varepsilon^{2} \widetilde{D}_{+},\left.\partial_{\lambda} D_{+}^{\varepsilon}\right|_{\lambda=\varepsilon=0} \neq 0$, we find a unique solution $\lambda_{0}^{+}(\varepsilon)$ of the equation $D_{+}^{\varepsilon}(\lambda)=0$ in the domain $|\varepsilon|<\varepsilon_{1}$ with some $\varepsilon_{1}>0$ that satisfies the condition $\lambda_{0}^{+}(0)=0$.

We compute the asymptotic formulas (1.17) and (1.18). From relations (1.8) and (2.5), we have

$$
T_{\nu}^{0}(\lambda)=1+\frac{\nu^{4}}{4 !} \lambda+O\left(\lambda^{2}\right), \quad \eta_{\nu}(\lambda)=v_{\nu}+O(\lambda), \quad|\lambda| \rightarrow 0, \quad \nu=1,2,
$$

where $v_{\nu}$ is defined in (1.19). Let $\lambda=r_{0}^{-}(\varepsilon)$. Relation (1.8) implies that $\rho^{0}(\lambda)=$ $\frac{\lambda}{4}+O\left(\lambda^{2}\right), \varepsilon \rightarrow 0$. Substituting this asymptotic relation in the first relation in (6.2), 
we get $0=\rho^{\varepsilon}(\lambda)=\frac{\lambda}{4}+O\left(\lambda^{2}\right)+O\left(\varepsilon^{2}\right), \varepsilon \rightarrow 0$, whence $\lambda=O\left(\varepsilon^{2}\right)$. Therefore, $\rho^{0}(\lambda)=$ $\frac{\lambda}{4}+O\left(\varepsilon^{4}\right)$ and, substituting (6.4) in the second asymptotic relation in (6.2), we get $\widetilde{\rho}(\lambda, \varepsilon)=\frac{v_{2}}{2}-2 v_{1}+O(\varepsilon), \varepsilon \rightarrow 0$. Again, substituting these asymptotic relations in the first relation in (6.2), we see that

$$
0=\rho^{\varepsilon}(\lambda)=\frac{\lambda}{4}+\varepsilon^{2}\left(\frac{v_{2}}{2}-2 v_{1}\right)+O\left(\varepsilon^{3}\right), \quad \lambda=r_{0}^{-}(\varepsilon), \text { as } \varepsilon \rightarrow 0,
$$

which yields the first asymptotic relation in (1.17).

Let $\lambda=\lambda_{0}^{+}(\varepsilon)$. Relations (1.8) show that $D_{+}^{0}(\lambda)=-\frac{\lambda}{4}+O\left(\lambda^{2}\right), \varepsilon \rightarrow 0$. The first relation in (6.3) yields $0=D_{+}^{\varepsilon}(\lambda)=-\frac{\lambda}{4}+O\left(\lambda^{2}\right)+O\left(\varepsilon^{2}\right)$, whence $\lambda=O\left(\varepsilon^{2}\right)$. Then $D_{+}^{0}(\lambda)=-\frac{\lambda}{4}+O\left(\varepsilon^{4}\right)$. Substituting (6.4) in the second asymptotic formula in (6.3), we have $\widetilde{D}_{+}(\lambda, \varepsilon)=2 v_{1}-\frac{v_{2}}{2}+O(\varepsilon)$. Substituting these asymptotic formulas in the first relation in (6.3), we get

$$
0=D_{+}^{\varepsilon}(\lambda)=-\frac{\lambda}{4}+\varepsilon^{2}\left(2 v_{1}-\frac{v_{2}}{2}\right)+O\left(\varepsilon^{3}\right), \quad \lambda=\lambda_{0}^{+}(\varepsilon),
$$

whence the second asymptotic relation in (1.17) follows.

We prove (1.18). From the asymptotic relation (1.17) and (6.2), it follows that

$$
\rho^{\varepsilon}\left(\lambda_{0}^{+}\right)=s y(\varepsilon), \quad y(\varepsilon)=\left(\rho^{\varepsilon}\right)^{\prime}\left(r_{0}^{-}\right)+O(s)=\left(\rho^{0}\right)^{\prime}\left(r_{0}^{-}\right)+O\left(\varepsilon^{2}\right)=\frac{1}{4}+O\left(\varepsilon^{2}\right)
$$

as $\varepsilon \rightarrow 0$, where $s=\lambda_{0}^{+}-r_{0}^{-} \rightarrow 0$. Substituting $\rho^{\varepsilon}\left(\lambda_{0}^{+}\right)=s y(\varepsilon)$ in the relation $D_{+}=\left(T_{1}-1\right)^{2}-\rho\left(\right.$ see $(3.15)$ ) and using the relation $D_{+}\left(\lambda_{0}^{+}\right)=0$, we obtain

$$
s=\lambda_{0}^{+}-r_{0}^{-}=\frac{\left(T_{1}^{\varepsilon}\left(\lambda_{0}^{+}\right)-1\right)^{2}}{y(\varepsilon)} .
$$

Substituting the asymptotic relation (1.17) in (6.1) and using (6.4), we have

$$
T_{1}^{\varepsilon}\left(\lambda_{0}^{+}\right)=1-\varepsilon^{2} A+O\left(\varepsilon^{3}\right), \quad A=\frac{v_{2}}{12}-\frac{4 v_{1}}{3} .
$$

Substituting (6.5) and (6.7) in (6.6), we derive the asymptotic relation (1.18).

The relations $\Delta_{\nu}^{\varepsilon}=T_{1}^{\varepsilon}-(-1)^{\nu} \sqrt{\rho^{\varepsilon}}, \nu=1,2$, imply that

$$
\Delta_{\nu}^{\varepsilon}(\lambda)=T_{1}^{\varepsilon}\left(r_{0}^{-}\right)-(-1)^{\nu} \sqrt{\lambda-r_{0}^{-}} \sqrt{y(\varepsilon)}+O\left(\left(\lambda-r_{0}^{-}\right)^{\frac{3}{2}}\right), \quad \lambda-r_{0}^{-} \rightarrow+0 .
$$

Therefore, the function $\Delta_{1}^{\varepsilon}$ is monotone increasing and the function $\Delta_{2}^{\varepsilon}$ is monotone decreasing in an interval $\left(r_{0}^{-}, r_{0}^{-}+\varepsilon\right), \varepsilon>0$ (see Figure 3). The asymptotics (1.18), (6.7) imply the asymptotics

$$
\Delta_{1}^{\varepsilon}\left(r_{0}^{-}\right)=\Delta_{2}^{\varepsilon}\left(r_{0}^{-}\right)=T_{1}^{\varepsilon}\left(r_{0}^{-}\right)=T_{1}^{\varepsilon}\left(\lambda_{0}^{+}\right)+O\left(\varepsilon^{4}\right)=1-\varepsilon^{2} A+O\left(\varepsilon^{3}\right)
$$

as $\varepsilon \rightarrow 0$. Suppose $A>0$. Then there exists $\delta>0$ such that $-1<\Delta_{1}^{\varepsilon}\left(r_{0}^{-}\right)<1$ for every $\varepsilon$ with $-\delta<\varepsilon<\delta$. Since $\Delta_{1}^{\varepsilon}$ is monotone increasing on $\left(r_{0}^{-}, r_{0}^{-}+\varepsilon\right)$, $\varepsilon>0$, from Theorem 1.1, iv) it follows that $\Delta_{1}^{\varepsilon}$ increases in the interval $\left(r_{0}^{-}, \lambda_{0}(\varepsilon)\right)$, where $\Delta_{1}^{\varepsilon}\left(\lambda_{0}(\varepsilon)\right)=1$. Therefore, $\lambda_{0}(\varepsilon)=\lambda_{2 n}^{ \pm}(\varepsilon)$ for some $n$. Note that $\lambda_{0}(0)=0$, because $\Delta_{1}^{0}(\lambda)=\cosh z$. Since $\lambda_{0}^{+}(0)=0$, we have $\lambda_{0}(\varepsilon)=\lambda_{0}^{+}(\varepsilon)$. Consequently, $-1<\Delta_{1}^{\varepsilon}<1$ on $\alpha=\left(r_{0}^{-}(\varepsilon), \lambda_{0}^{+}(\varepsilon)\right)$ and $\Delta_{1}^{\varepsilon}(\lambda)>1$ for $\lambda>\lambda_{0}^{+}$. Substituting the asymptotic relations (6.1) and (6.2) in the relations $\Delta_{2}^{\varepsilon}=T_{1}^{\varepsilon}-\sqrt{\rho^{\varepsilon}}$, we get $\Delta_{2}^{\varepsilon}=\cos z+o(\varepsilon), \varepsilon \rightarrow 0$. Therefore, the function $\Delta_{2}^{\varepsilon}+1,-\delta<\varepsilon<\delta$, has no zeros on the interval $\alpha$. Thus, $-1<\Delta_{2}^{\varepsilon}<1$ on $\alpha$. By Theorem 1.1, iii), the spectrum has multiplicity 4 in the interval $\alpha$ and multiplicity 2 outside this interval. 
It remains to prove that $A>0$. Using (1.19) and straightforward calculations, we arrive at the relation

$$
A=\frac{1}{4} \int_{0}^{1} f(s) \int_{s}^{1} p(t) p(t-s) d t d s, \quad f(s)=s(s-1) .
$$

We have

$$
f(t)=\sum_{n} f_{n} e^{i 2 \pi n t}, \quad f_{n}=\frac{2}{(2 \pi n)^{2}}, \quad n \neq 0, \quad f_{0}=-\frac{1}{6}, \quad p(t)=\sum_{n} \widehat{p}_{n} e^{i 2 \pi n t} .
$$

Substituting these relations in (6.8), we obtain

$$
A=\frac{1}{4} \sum_{m, n} \hat{p}_{n} \widehat{p}_{m} \int_{0}^{1} f(s) d s \int_{s}^{1} e^{i 2 \pi(n+m) t} e^{-i 2 \pi n s} d t=\frac{1}{4}\left(F_{1}+F_{2}\right),
$$

where

$$
\begin{aligned}
F_{2} & =\sum_{m+n \neq 0} \frac{\widehat{p}_{n} \hat{p}_{m}}{2 \pi i(n+m)} \int_{0}^{1} f(s) e^{-i 2 \pi n s}\left(1-e^{i 2 \pi(n+m) s}\right) d s \\
& =\sum_{m+n \neq 0} \hat{p}_{n} \widehat{p}_{m} \frac{f_{n}-f_{m}}{2 \pi i(n+m)}=0
\end{aligned}
$$

and

$$
F_{1}=\sum_{-\infty}^{\infty}\left|\widehat{p}_{n}\right|^{2} \int_{0}^{1} f(s)(1-s) e^{-i 2 \pi n s} d s
$$

Note that

$$
\int_{0}^{1} f(s)(1-s) e^{-i 2 \pi n s} d s=\sum_{k} \int_{0}^{1}(1-s) f_{k} e^{i 2 \pi(k-n) s} d s=\sum_{k \neq n} \frac{-f_{k}}{i 2 \pi(k-n)}+\frac{f_{n}}{2} .
$$

Then

$$
F_{1}=\sum_{n \neq 0}\left|\widehat{p}_{n}\right|^{2}\left(\sum_{k \neq n} \frac{-f_{k}}{i 2 \pi(k-n)}+\frac{f_{n}}{2}\right)=\sum_{n \neq 0}\left|\hat{p}_{n}\right|^{2} \frac{f_{n}}{2}>0,
$$

because $\hat{p}_{0}=0, F_{1}$ is real, and $f_{k}>0, k \neq 0$. Substituting (6.11) and (6.12) in (6.10) and using the expression for $f_{n}$ from (6.9), we derive the second relation for $A$ in (1.18), which implies that $A>0$.

\section{§7. Appendix}

In terms of the functions

$$
f_{j}=z^{-2} e^{-z t \omega_{j}} \vartheta_{j}, \quad j \in \mathbb{N}_{3},
$$

equation (3.3) takes the form

$$
\begin{gathered}
f_{j}=v_{j}+\frac{1}{4 z} \sum_{n=0}^{3} \omega_{n} v_{n} h_{n j}, \quad \text { where } \quad v_{j}=\omega_{j}^{2} p+\frac{\omega_{j} p^{\prime}}{z}+\frac{q}{z^{2}}, \\
h_{k j}=E_{k j} f_{j}, \quad\left(E_{k j} f\right)(t, \lambda)=\int_{0}^{1} e_{k j}(t-s, \lambda) f(s) d s, \quad(t, \lambda) \in \mathbb{R} \times \mathbb{C}, \\
e_{k j}(t, \lambda)=\left\{\begin{array}{ll}
e^{z t\left(\omega_{k}-\omega_{j}\right)} \chi(-t) & \text { if } k<j, \\
-e^{z t\left(\omega_{k}-\omega_{j}\right)} \chi(t) & \text { if } k \geq j,
\end{array} \quad \chi(t)= \begin{cases}0 & \text { if } t<0, \\
1 & \text { if } t \geq 0 .\end{cases} \right.
\end{gathered}
$$


If $(k, j, t) \in \mathbb{N}_{3}^{2} \times \mathbb{R}$ and $(j-k) t \geq 0$, then $\left|e^{z t \omega_{j}}\right|=e^{t \operatorname{Re}\left(z \omega_{j}\right)} \leq e^{t \operatorname{Re}\left(z \omega_{k}\right)}$. Consequently,

$$
\left|e_{k j}(t, \lambda)\right| \leq 1, \quad(k, j, t, \lambda) \in \mathbb{N}_{3}^{2} \times \mathbb{R} \times \mathbb{C} .
$$

Lemma 7.1. For every $(j, \lambda) \in \mathbb{N}_{3} \times \Lambda_{1}$, the integral equation (7.2) has a unique solution $f_{j}(\cdot, \lambda) \in L^{1}(0,1)$. Every function $f_{j}(t, \cdot), t \in[0,1]$, is analytic in $\Lambda_{1}^{ \pm}$and satisfies the inequalities

$$
\left\|f_{j}(\cdot, \lambda)\right\| \leq 2 \varkappa, \quad(j, \lambda) \in \mathbb{N}_{3} \times \Lambda_{2} .
$$

Proof. Iterations in (7.2) provide the relations

$$
f_{j}=\sum_{n=0}^{\infty} f_{j, n}, \quad f_{j, 0}=v_{j}, \quad f_{j, n}(t, \lambda)=\frac{1}{4 z} \int_{0}^{1} K_{j}(t, s, \lambda) f_{j, n-1}(s, \lambda) d s,
$$

where $n \geq 1, K_{j}(t, s, \lambda)=\sum_{0}^{3} \omega_{i} v_{i}(t, \lambda) e_{i j}(t-s, \lambda)$. From relations (7.7) we have

$$
f_{j, n}(t, \lambda)=\frac{1}{(4 z)^{n}} \int_{[0,1]^{n}} K_{j}\left(t, t_{n}, \lambda\right) K_{j}\left(t_{n}, t_{n-1}, \lambda\right) \cdots K_{j}\left(t_{2}, t_{1}, \lambda\right) f_{j, 0}\left(t_{1}, \lambda\right) d t_{1} \cdots d t_{n} .
$$

Using (7.5), we get

$$
\max \left\{\frac{1}{4}\left|K_{j}(t, s, \lambda)\right|,\left|f_{j, 0}(t, \lambda)\right|\right\} \leq|p(t)|+\frac{\left|p^{\prime}(t)\right|}{|z|}+\frac{|q(t)|}{|z|^{2}}, \quad(t, s, \lambda) \in[0,1]^{2} \times \mathbb{C},
$$

and

$$
\begin{aligned}
\left\|f_{j, n}(\cdot, \lambda)\right\| \leq & \frac{1}{|4 z|^{n}} \int_{[0,1]^{n+1}}\left|K_{j}\left(t, t_{n}, \lambda\right) \| K_{j}\left(t_{n}, t_{n-1}, \lambda\right)\right| \cdots \\
& \quad \times\left|K_{j}\left(t_{2}, t_{1}, \lambda\right)\right|\left|f_{j, 0}\left(t_{1}, \lambda\right)\right| d t_{1} \cdots d t_{n} d t \\
\leq & \frac{1}{|z|^{n}}\left(\|p\|+\frac{\left\|p^{\prime}\right\|}{|z|}+\frac{\|q\|}{|z|^{2}}\right)^{n+1} \leq \frac{\varkappa^{n+1}}{|z|^{n}} \quad \text { as } \quad|z|>1 .
\end{aligned}
$$

These estimates show that for every fixed $\lambda \in \Lambda_{1}$, the series (7.7) converges absolutely and uniformly on the interval $[0,1]$. Consequently, the sum of this series provides a unique solution of equation (7.2). For every $t \in \mathbb{R}$, the series (7.7) converges absolutely and uniformly on any compact set in $\Lambda_{1}$. Each term of this series is an analytic function of the variable $\lambda$ in $\Lambda_{1}^{ \pm}$. Therefore, for every fixed $t \in[0,1]$ the function $f_{j}(t, \cdot)$ is analytic at $\lambda \in \Lambda_{1}^{ \pm}$. Summing the majorants, we get $\left\|f_{j}\right\| \leq \frac{\varkappa}{1-\frac{\varkappa}{|z|}}$, which implies (7.6).

Proof of Lemma 3.1. i) The matrix $\mathcal{M}(t, \lambda)=\left(\varphi_{j}^{(k)}(t, \lambda)\right)_{k, j=0}^{3},(t, \lambda) \in[0,1] \times \mathbb{C}$, satisfies the relations

$$
\mathcal{M}^{\prime}=\left(\begin{array}{cccc}
0 & 1 & 0 & 0 \\
0 & 0 & 1 & 0 \\
0 & 0 & 0 & 1 \\
\lambda-q & -p^{\prime} & -p & 0
\end{array}\right) \mathcal{M}, \quad \mathcal{M}(0, \lambda)=I_{4} .
$$

The matrix $\Theta$ is also a solution of equation (7.9), and thus $\mathcal{M}(t, \cdot)=\Theta_{t} \Theta_{0}^{-1}$ for all $t \in[0,1]$. The relation $M=\mathcal{M}(1, \cdot)$ implies (3.5). From (7.1) and Lemma 7.1 it follows that $\Theta$ is analytic in $\Lambda_{1}^{ \pm}$; thus, $\Phi$ is also analytic.

We prove (3.8). Consider the matrices

$$
Z=\left(\delta_{k j} z^{k}\right)_{k, j=0}^{3}, \quad B_{t}=\left(b_{k j}(t, \cdot)\right)_{k, j=0}^{3}=Z^{-1} \Theta_{t} e^{-z t \Omega}, \quad b_{k j}=z^{-k} \vartheta_{j}^{(k)} e^{-z t \omega_{j}} .
$$

From (3.3) we have $b_{k j}=\omega_{j}^{k}+\frac{1}{4 z} \sum_{n=0}^{3} \omega_{n}^{k+1} h_{n j}$, where the $h_{n j}$ are defined in (7.2). This implies that

$$
B_{t}=X+\frac{X \Omega A_{t}}{4 z}, \quad X=\left(\omega_{j}^{k}\right)_{k, j=0}^{3}, \quad A_{t}=\left(h_{k j}(t, \cdot)\right)_{k, j=0}^{3} .
$$


Relations (7.3) and estimates (7.5) and (7.6) show that $A_{0}(\lambda)$ is bounded as $|\lambda| \rightarrow \infty$; then $B_{0}(\lambda)=X+O\left(\frac{1}{z}\right)$. The relations $\Theta_{0}=Z B_{0}$ (see (7.10) ) yield (3.8).

We prove inequalities (3.6). Substituting the relations for $B_{t}$ from (17.10) in the relation for $\Phi$ from (3.5), we obtain

$$
\Phi=B_{0}^{-1} B_{1}=\left(X^{-1} B_{0}\right)^{-1} X^{-1} B_{1}=\left(I_{4}+\frac{\Omega A_{0}}{4 z}\right)^{-1}\left(I_{4}+\frac{\Omega A_{1}}{4 z}\right) .
$$

Substituting (7.4) in (7.3), we have

$$
h_{k j}(0, \lambda)=0, \quad k \geq j, \quad h_{k j}(1, \lambda)=0, \quad k<j .
$$

Therefore, $\left(\Omega A_{0}\right)^{4}=0$ and $\left(I_{4}+\frac{\Omega A_{0}}{4 z}\right)^{-1}=\sum_{0}^{3}(-1)^{n}\left(\frac{\Omega A_{0}}{4 z}\right)^{n}$. Then

$$
\Phi=I_{4}+\left(\frac{\Omega}{4 z}-\frac{\Omega A_{0} \Omega}{(4 z)^{2}}+\frac{\left(\Omega A_{0}\right)^{2} \Omega}{(4 z)^{3}}\right)\left(A_{1}-A_{0}\right)-\frac{\left(\Omega A_{0}\right)^{3} \Omega A_{1}}{(4 z)^{4}} .
$$

From (7.12) we get

$h_{k j}(1, \lambda)-h_{k j}(0, \lambda)=h_{k j}\left(\chi_{k-j}, \lambda\right) s_{k-j}, \quad \chi_{n}=\left\{\begin{array}{ll}0 & \text { if } n<0, \\ 1 & \text { if } n \geq 0,\end{array} \quad s_{n}= \begin{cases}-1 & \text { if } n<0 \\ 1 \quad \text { if } n \geq 0\end{cases}\right.$ whence for $\lambda \in \Lambda_{1}$ we have

$$
\begin{aligned}
\phi_{k j}(\lambda)= & \delta_{k j}+\frac{\omega_{k}}{4 z} h_{k j}\left(\chi_{k-j}, \lambda\right) s_{k-j}-\frac{\omega_{k}}{(4 z)^{2}} \sum_{k<r \leq 3} \omega_{r} h_{k r}(0, \lambda) h_{r j}\left(\chi_{r-j}, \lambda\right) s_{r-j} \\
& +\frac{\omega_{k}}{(4 z)^{3}} \sum_{k<n<r \leq 3} \omega_{r} \omega_{n} h_{k n}(0, \lambda) h_{n r}(0, \lambda) h_{r j}\left(\chi_{r-j}, \lambda\right) s_{r-j} \\
& -\frac{\omega_{3} h_{3 j}(1, \lambda)}{(4 z)^{4}} \prod_{0 \leq r \leq 2} \omega_{r} h_{r, r+1}(0, \lambda) .
\end{aligned}
$$

Estimates (7.5) and (7.6) imply that $\left\|h_{n j}(\cdot, \lambda)\right\| \leq 2 \varkappa, \lambda \in \Lambda_{2}$. Then, by (7.13),

$$
\left|\phi_{k j}(\lambda)-\delta_{k j}\right| \leq \frac{\varkappa}{2 z}+(3-k)\left(\frac{\varkappa}{2 z}\right)^{2}+\frac{(4-k)(3-k)}{2}\left(\frac{\varkappa}{2 z}\right)^{3}+\left(\frac{\varkappa}{2 z}\right)^{4}
$$

Since $|z| \geq 3 \varkappa$ for $\lambda \in \Lambda_{3}^{ \pm}$, we have the second inequality in (3.6). Next, $\left|\phi_{j j}(\lambda)\right| \leq$ $1+\left|\phi_{j j}(\lambda)-1\right| \leq 1+\frac{\varkappa}{|z|}$, which yields the first inequality in (3.6).

We prove (3.7). If $f^{\prime} \in L^{1}(0,1)$, then integration by parts gives the relation

$$
\left(E_{k j} f\right)(t, \lambda)=\frac{1}{z\left(\omega_{k}-\omega_{j}\right)}\left(f(t)-e^{z\left(\omega_{k}-\omega_{j}\right)\left(t-\chi_{j-k}\right)} f\left(\chi_{j-k}\right)+\left(E_{k j} f^{\prime}\right)(t, \lambda)\right)
$$

for $k \neq j$. Using (7.14), from (17.3) we obtain $h_{k j}(t, \lambda)=O\left(z^{-1}\right)$ uniformly with respect to $t \in[0,1]$ as $|\lambda| \rightarrow \infty, k \neq j$. By (17.13), we have

$$
\phi_{k j}(\lambda)=\delta_{k j}+\frac{\omega_{k}}{4 z} h_{k j}\left(\chi_{k-j}, \lambda\right) s_{k-j}+O\left(z^{-4}\right) \text { as }|\lambda| \rightarrow \infty, \quad k, j \in \mathbb{N}_{3} .
$$

This implies the first asymptotic relation in (3.7). We prove the second asymptotic relation in (3.7). From relations (7.2)-(7.4), it follows that

$$
h_{j j}(1, \lambda)=-\int_{0}^{1} f_{j}(t, \lambda) d t=-\omega_{j}^{2} \widehat{p}_{0}+\frac{\omega_{j} \widehat{p}_{0}^{2}}{8 z}+O\left(z^{-2}\right) \text { as }|\lambda| \rightarrow \infty .
$$

Substituting this asymptotic formula in (7.15), we get

$$
\phi_{j j}(\lambda)=1+\frac{\omega_{j}}{4 z} h_{j j}(1, \lambda)+O\left(z^{-4}\right)=1-\frac{\omega_{j}^{3} \widehat{p}_{0}}{4 z}+\frac{\omega_{j}^{2} \widehat{p}_{0}^{2}}{32 z^{2}}+O\left(z^{-3}\right),
$$

which provides the second asymptotic formula in (3.7).

ii) Relations (7.2) imply that

$$
f_{j}(t, \lambda)=\omega_{j}^{2} p(t)+\frac{\omega_{j}}{z} p^{\prime}(t)+\frac{\omega_{j}^{3}}{4 z} p(t) h_{j j}(t, \lambda)+O\left(z^{-2}\right) \text { as }|\lambda| \rightarrow \infty
$$


uniformly with respect to $t \in[0,1]$. Substituting this asymptotic relation in (7.3) and using (7.14), we get

$$
h_{k j}(t, \lambda)=\omega_{j}^{2}\left(E_{k j} p\right)(t, \lambda)+\frac{\omega_{j}}{z}\left(E_{k j} p^{\prime}\right)(t, \lambda)+O\left(z^{-2}\right) \text { as }|\lambda| \rightarrow \infty, \quad k \neq j,
$$

uniformly with respect to $t \in[0,1]$. Next, we prove (3.10). The proof of (3.9) is similar. Let $z=(1+i) \pi n+O\left(n^{-1}\right), n \rightarrow \infty$. Then from (17.16) it follows that

$$
h_{01}(0, \lambda)=\omega_{1}^{2}\left(E_{01} p\right)(0, \lambda)+\frac{\omega_{1}}{z}\left(E_{01} p^{\prime}\right)(0, \lambda)+O\left(n^{-2}\right) .
$$

Applying (7.14) and the asymptotic formula $z\left(\omega_{0}-\omega_{1}\right)=z(1+i)=-2 i \pi n+O\left(n^{-1}\right)$, we obtain

$$
h_{01}(0, \lambda)=\frac{\omega_{0} \omega_{1}\left(E_{01} p^{\prime}\right)(0, \lambda)}{z\left(\omega_{0}-\omega_{1}\right)}+O\left(n^{-2}\right)=-2 \xi \widehat{p_{n}^{\prime}}+O\left(\xi^{2}\right), \quad \xi=\frac{1}{4 \pi n} .
$$

Substituting this in (7.15), we get $\phi_{01}(\lambda)=(1-i) \xi^{2} \widehat{p_{n}^{\prime}}+O\left(\xi^{3}\right)$ for $z=(1+i) \pi n+O\left(n^{-1}\right)$, $n \rightarrow \infty$. Similar arguments lead to the relation $\phi_{10}(\lambda)=(1+i) \xi^{2} \widehat{\widehat{p_{n}^{\prime}}}+O\left(\xi^{3}\right)$, which implies (3.10).

\section{REFERENCES}

[1] A. Badanin and E. Korotyaev, Spectral asymptotics for periodic fourth-order operators, Int. Math. Res. Not. 2005, no. 45, 2775-2814. MR2182471 (2006f:34064)

[2] A. Badanin, J. Brüning, and E. Korotyaev, The Lyapunov function for Schrödinger operators with a periodic $2 \times 2$ matrix potential, J. Funct. Anal. 234 (2006), no. 1, 106-126. MR2214141 (2006k:47090)

[3] R. Carlson, Eigenvalue estimates and trace formulas for the matrix Hill's equation, J. Differential Equations 167 (2000), no. 1, 211-244. MR1785119 (2001e:34157)

[4] _ A spectral transform for the matrix Hill's equation, Rocky Mountain J. Math. 34 (2004), no. 3, 869-895. MR2087436 (2005e:34013)

[5] D. Chelkak and E. Korotyaev, Spectral estimates for Schrödinger operators with periodic matrix potentials on the real line, Int. Math. Res. Not. 2006, Art. ID 60314, 41 pp. MR 2219217(2007g:47071)

[6] S. Clark and F. Gesztesy, Weyl-Titchmarsh $M$-function asymptotics, local uniqueness results, trace formulas, and Borg-type theorems for Dirac operators, Trans. Amer. Math. Soc. 354 (2002), no. 9, 3475-3534. MR1911509 (2003i:34191)

[7] S. Clark, F. Gesztesy, H. Holden, and B. Levitan, Borg-type theorems for matrix-valued Schrödinger and Dirac operators, J. Differential Equations 167 (2000), 181-210. MR1785118 (2002d:34019)

[8] N. Dunford and J. T. Schwartz, Linear operators. Pt. II. Spectral theory. Selfadjoint operators in Hilbert space, Intersci. Publ. John Wiley and Sons, Inc., New York-London, 1963. MR0188745 $(32: 6181)$

[9] B. A. Dubrovin, I. M. Krichever, and S. P. Novikov, Integrable systems. I, Dynamical Systems. IV, Itogi Nauki i Tekhniki Sovrem. Probl. Mat. Fundam. Naprav., vol. 4, VINITI, Moscow, 1985, pp. 179-285; English transl., Encyclopaedia Math. Sci., vol. 4, Springer-Verlag, Berlin-New York, 1990, pp. 173-280. MR0842910 (87k:58112)

[10] I. M. Gel'fand and V. B. Lidskiǔ, On the structure of the regions of stability of linear canonical systems of differential equations with periodic coefficients, Uspekhi Mat. Nauk 10 (1955), no. 1, 3-40. (Russian) MR0073767 (17:482g)

[11] J. Hoppe, A. Laptev, and J. Ostensson, Solitons and the removal of eigenvalues for fourth-order differential operators, Int. Math. Res. Not. 2006, Art ID 85050, 14 pp. MR.2233712 (2007i:34137)

[12] T. Kato, Perturbation theory for linear operators, Classics in Math., Springer-Verlag, Berlin, 1995. MR 1335452 (96a:47025)

[13] E. Korotyaev, Spectral estimates for matrix-valued periodic Dirac operators, Asymptot. Anal. 59 (2008), no. 3-4, 195-225. MR2450359 (2009m:34202)

[14] _ Inverse resonance scattering on the real line, Inverse Problems 21 (2005), 325-341. MR 2146179(2006a:34033)

[15] , Conformal spectral theory for the monodromy matrix, Trans. Amer. Math. Soc. 362 (2010), 3435-3462. MR2601596

[16] E. Korotyaev and A. Kutsenko, Borg-type uniqueness theorems for periodic Jacobi operators with matrix-valued coefficients, Proc. Amer. Math. Soc. 137 (2009), 1989-1996. MR2480280 (2010j:47047) 
[17] _ Lyapunov functions of periodic matrix-valued Jacobi operators, Spectral Theory of Differential Operators, Amer. Math. Soc. Transl. Ser. 2, vol. 225, Amer. Math. Soc., Providence, RI, 2008, pp. 117-131. MR2509779(2010i:47062)

[18] E. Korotyaev and I. Lobanov, Schrödinger operators on zigzag nanotubes, Ann. Henri Poincaré 8 (2007), 1151-1176. MR2355344 (2008g:81076)

[19] M. G. Kreın, The basic propositions of the theory of $\lambda$-zones of stability of a canonical system of linear differential equations with periodic coefficients, In Memory of Aleksandr Aleksandrovich Andronov, Akad. Nauk SSSR, Moscow, 1955. (Russian) MR0075382 (17:738c)

[20] H. McKean, Boussinesq's equation on the circle, Comm. Pure Appl. Math. 34 (1981), 599-691. MR0622617 (82j:58063)

[21] V. Mikhailets and V. Molyboga, Singular eigenvalue problems on the circle, Methods Funct. Anal. Topology 10 (2004), no. 3, 44-53. MR2092532(2005e:34255)

[22] __ Uniform estimates for the semi-periodic eigenvalues of the singular differential operators, Methods Funct. Anal. Topology 10 (2004), no. 4, 30-57. MR2109216 (2005h:34221)

[23] F. G. Maksudov and O. A. Veliev, Spectral analysis of differential operators with periodic matrix coefficients, Differentsial'nye Uravneniya 25 (1989), no. 3, 400-409; English transl., Differential Equations 25 (1989), no. 3, 271-277. MR0994320(90d:47045)

[24] V. A. Marchenko, Sturm-Liouville operators and their applications, Naukova Dumka, Kiev, 1977; English transl., Oper. Theory Adv. Appl., vol. 22, Birkhäuser Verlag, Basel, 1986. MR0481179 (58:1317) MR0897106 (88f:34034)

[25] M. A. Naı̆mark, Linear differential operators, 2nd ed.. Nauka, Moscow, 1969; English transl. of 1st ed., Frederick Ungar Publ. Co., New York, Pt. 1, 1967; Pt. 2, 1968. MR0353061 (50:5547) MR0216050(35:6885) MR0262880(41:7485)

[26] V. G. Papanicolaou, The spectral theory of the vibrating periodic beam, Comm. Math. Phys. 170 (1995), 359-373. MR1334400 (96d:34108)

[27] _ The periodic Euler-Bernoulli equation, Trans. Amer. Math. Soc. 355 (2003), no. 9, 37273759. MR 1990171 (2004c:34041)

[28] V. G. Papanicolaou and D. Kravvaritis, The Floquet theory of the periodic Euler-Bernoulli equation, J. Differential Equations 150 (1998), 24-41. MR1660270 (2000a:34167)

[29] J. Pöschel and E. Trubowitz, Inverse spectral theory, Pure Appl. Math., vol. 130, Acad. Press, Boston, MA, 1987. MR0894477 (89b:34061)

[30] M. Reed and B. Simon, Methods of modern mathematical physics. IV. Analysis of operators, Acad. Press, New York-London, 1978. MR0493421 (58:12429c)

[31] V. Tkachenko, Spectrum of 1-d selfadjoint periodic differential operator of order 4, Advances in Differential Equations and Mathematical Physics (Birmingham, AL, 2002), Contemp. Math., vol. 327, Amer. Math. Soc., Providence, RI, 2003, pp. 331-340. MR.1991552 (2004f:47066)

[32] _ Expansions associated with 1-d periodic differential operators of order 4, Recent Advances in Differential Equations and Mathematical Physics, Contemp. Math., vol. 412, Amer. Math. Soc., Providence, RI, 2006, pp. 283-296. MR2259115 (2007i:34140)

[33] __ Eigenfunction expansions associated with one-dimensional periodic differential operators of order 2n, Funktsional. Anal. i Prilozhen. 41 (2007), no. 1, 66-89; English transl., Funct. Anal. Appl. 41 (2007), no. 1, 54-72. MR2333983(2008e:34202)

[34] V. A. Yakubovich and V. M. Starzhinskiı̌, Linear differential equations with periodic coefficients and their applications, Nauka, Moscow, 1972; English transl., Halsted Press, New York-Toronto, 1975. MR0364739 (51:993) MR0364740 (51:994)

[35] M. Zworski, Distribution of poles for scattering on the real line, J. Funct. Anal. 73 (1987), 277-296. MR0899652(88h:81223)

Northern (Arctic) Federal University, Northern Dvina Quay 17, Archangelsk, Russia E-mail address: an.badanin@gmail.com

St. Petersburg State University, Ul'yanovskaya 3, Petrodvoretz, St. Petersburg 198504, Russia, and Leningrad State University named after A. S. Pushkin, Peterburgskoe Shosse 10, Pushinin, St. Petersburg 196605, Russia

E-mail address: korotyaev@gmail.com

Received 11/MAR/2009

Translated by N. B. LEBEDINSKAYA 